NSF-KITP-06-97

MIT-CTP-3798

BRX TH-578

UPR-1176-T

\title{
Torsion and Supersymmetry Breaking
}

\author{
Albion Lawrence ${ }^{1}$, Tobias Sander ${ }^{1}$, \\ Michael B. Schulz ${ }^{2,3}$, and Brian Wecht ${ }^{4,5}$ \\ 1 Theory Group, Martin Fisher School of Physics, Brandeis University, \\ MS 057, PO Box 549110, Waltham, MA 02454, USA \\ 2 Department of Physics, Bryn Mawr College, Bryn Mawr, PA 19010, USA
}

3 Department of Physics and Astronomy, University of Pennsylvania, Philadelphia, PA 19104, USA

4 Center for Theoretical Physics, MIT, Cambridge, MA 02139, USA

5 School of Natural Sciences, Institute for Advanced Study, Princeton, NJ 08450, USA

We identify the auxiliary fields in the hypermultiplets of type IIB string theory compactified on a Calabi-Yau manifold, using a combination of worldsheet and supergravity techniques. The SUSY-breaking squark and gaugino masses in type IIB models depend on these auxiliary fields, which parametrize deformations away from a pure Calabi-Yau compactification to one with NS-NS 3-form flux and $S U(3) \times S U(3)$ structure. Worldsheet arguments show that such compactifications are generically globally nongeometric. Our results, combined with earlier results for type IIA compactifications, imply that these deformations are the mirrors of NS-NS 3-form flux, in accord with work from the supergravity point of view. Using the worldsheet current algebra, we explain why mirror symmetry may continue to hold in the presence of fluxes breaking the symmetries (e.g., (2,2) SUSY) on which mirror symmetry is typically taken to depend. Finally, we give evidence that nonperturbative worldsheet effects (such as worldsheet instantons) provide important corrections to the supergravity picture in the presence of auxiliary fields for Kähler moduli.

14 November 2007 


\section{Contents}

1. Introduction . . . . . . . . . . . . . . . . . . . . . . . . . . . . . . . . . 1

2. Review . . . . . . . . . . . . . . . . . . . . . . . . . 4

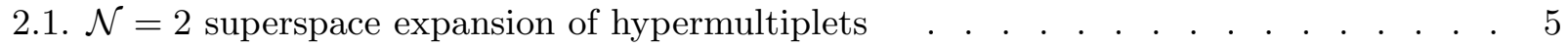

2.2. Spacetime supersymmetry and $G$-structures . . . . . . . . . . . . . . . . . . 7

2.3. Worldsheet vs. spacetime supersymmetry . . . . . . . . . . . . . . . . . . . 10

2.4. Review of $S U(3)$ structure . . . . . . . . . . . . . . . . . . . . . . . . . . 12

2.5. Review of $S U(3) \times S U(3)$ structure . . . . . . . . . . . . . . . . . . . . . . 14

3. Worldsheet vertex operators for auxiliary fields . . . . . . . . . . . . . . . . . . 15

3.1. Worldsheet supersymmetry and target space geometry . . . . . . . . . . . . . 15

3.2. Vertex operators for auxiliary fields . . . . . . . . . . . . . . . . . . . . . . 15

3.3. Target space interpretation . . . . . . . . . . . . . . . . . . . . . . . . . 17

3.4. $S U(3)$ structure compactifications . . . . . . . . . . . . . . . . . . . . . . 22

3.5. Nongeometric compactifications . . . . . . . . . . . . . . . . . . . . . . . 22

3.6. Mirror symmetry . . . . . . . . . . . . . . . . . . . . . . . . . . . 23

4. Ten-dimensional supergravity calculation of auxiliary fields . . . . . . . . . . . . . 25

4.1. Auxiliary field for the volume deformation $y_{1} \quad$. . . . . . . . . . . . . . . . . . 25

4.2. Auxiliary fields $y_{\mathbf{8}}$ for remaining Kähler moduli . . . . . . . . . . . . . . . . 26

4.3. Auxiliary field $y_{\phi}$ for the universal hypermultiplet . . . . . . . . . . . . . . . 27

5. Four-dimensional Supergravity calculation of auxiliary fields . . . . . . . . . . . . . 27

5.1. Superpotential for $S U(3)$ structure compactifications . . . . . . . . . . . . . . 28

5.2. Overall volume modulus: 1 of $S U(3)$. . . . . . . . . . . . . . . . . . . . . . . . 30

5.3. Primitive Kähler moduli: 8 of $S U(3)$. . . . . . . . . . . . . . . . . . . . . 31

5.4. Universal hypermultiplet . . . . . . . . . . . . . . . . . . . . . . . . . . 31

5.5. Complex structure moduli: 6 of $S U(3)$. . . . . . . . . . . . . . . . . . . . 32

6. Worldsheet instanton corrections . . . . . . . . . . . . . . . . . . . . . . . . . 32

6.1. General remarks . . . . . . . . . . . . . . . . . . . . . . . . . . . . . . 32

6.2. One-instanton contribution to the superpotential . . . . . . . . . . . . . . . . 33

7. Conclusions . . . . . . . . . . . . . . . . . . . . . . . . . . . . . . . . . . 35

Appendix A. Mathematical conventions . . . . . . . . . . . . . . . . . . . . . . . 36

A.1. Spinor conventions . . . . . . . . . . . . . . . . . . . . . . . . . . . . . 36

A.2. Metrics, $p$-forms, and the almost complex structure . . . . . . . . . . . . . . 38

\section{Introduction}

In this article, we compute the auxiliary fields of $\mathcal{N}=2$ hypermultiplets in type IIB Calabi-Yau compactifications to four dimensions. In type IIB, these multiplets contain the Kähler moduli and the dilaton-axion, as well as the RR axions. This work is a continuation of the program begun in [1, 2], which focused on the vector multiplets.

Our work has several motivations. The first, stemming from particle physics model building considerations, is that expectation values for these auxiliary fields generate explicit 
SUSY-breaking terms in the low energy four dimensional theory [3]. This has proven useful for understanding $\mathcal{N}=1$ flux compactifications. For example, it was shown in [1,2, 4 that the flux-induced superpotential $W=\int G_{3} \wedge \Omega$ derived in [5, [6, (7) can be computed as a term explicitly breaking $\mathcal{N}=2$ to $\mathcal{N}=1$ supersymmetry, proportional to the expectation values of auxiliary fields in vector multiplets.

The same computations are also useful in studying $\mathcal{N}=1$ to $\mathcal{N}=0$ supersymmetry breaking at lower energies in models with D-branes and/or fluxes. If $\mathcal{N}=2$ supersymmetry is broken at a higher scale than $\mathcal{N}=1$, as happens in many flux compactifications, and $N=1$ supersymmetry is broken at a still lower scale, one can separate the auxiliary fields into those (call them $F_{\text {high }}$ ) whose expectation values break $\mathcal{N}=2$ to $\mathcal{N}=1$, and those (call them $F_{\text {low }}$ ) whose expectation values break $\mathcal{N}=1$ to $\mathcal{N}=0$. After performing the necessary orientifold projections compatible with the $\mathcal{N}=1$ supersymmetry, our results should allow the closed string fields to be written as $\mathcal{N}=1$ superfields with auxiliary fields of type $F_{\text {low }}$. The auxiliary fields in these supermultiplets then parametrize supersymmetry breaking in the low-energy $\mathcal{N}=1$ effective Lagrangian. In particular, the SUSY-breaking squark and gaugino masses will depend on the auxiliary fields we compute here [1],2]. In the conclusions we will discuss some recent work on building SUSY-breaking string models, for which the results here and in [1,2] have some relevance.

A second motivation for our work arises from the desire to extend the powerful results of mirror symmetry to compactifications with $\mathcal{N}<2$ spacetime supersymmetry. For type II compactifications these involve NS-NS fluxes, and finding the mirrors of compactifications with such fluxes is a long-standing problem. However, in the cases that we can understand a compactification with $\mathcal{N}<2$ supersymmetry as a deformation of a $\mathcal{N}=2$ compactification by expectation values for auxiliary fields, we can make progress by understanding the action of the mirror map on these auxiliary fields.

More precisely, the auxiliary fields for hypermultiplets in type IIA (whose scalar components include the complex structure moduli) have been identified in [1,2] as a combination of NS-NS 3-form flux and a subset of the $S U(3)$ intrinsic torsion classes. These torsion classes parametrize deformations of the compactification away from a pure special holonomy compactification. The mirrors of these fluxes and torsion classes should be the auxiliary fields for the type IIB hypermultiplets, which include the Kähler moduli.

${ }^{1}$ In the notation of [8], the relevant torsion classes are $W_{3}$ and $W_{4}$, defined in Sec. 2.4 below. 
Supergravity arguments already suggest an answer. The mirrors of type IIA compactifications with purely electric日 NS-NS flux in $H^{(2,1)}(X) \oplus H^{(1,2)}(X)$ and intrinsic torsion of type $W_{3,4}$, have been identified with "half-flat" manifolds [9-17]. For more general NS-NS flux, the mirrors have been identified with compactifications of $S U(3) \times S U(3)$ structure 10,11, 18, 3 On the other hand, considerations of the effective four-dimensional superpotential [1924] and of the action of T-duality transformations (such as mirror symmetry) on NS-NS flux [25-29] indicate that these mirrors should be generically nongeometric in the sense discussed in [30] (other related approaches to nongeometric backgrounds include asymmetric orbifolds [31-35] and Landau-Ginzburg models [36]). One goal of the present work is to make this claim more precise, and to relate auxiliary fields in IIB hypermultiplets to (nongeometric) intrinsic torsions in $S U(3) \times S U(3)$ structure compactifications.

Our basic approach uses an $\mathcal{N}=2$ superspace formalism that is natural from the worldsheet point of view. Our computations confirm both lessons of the previous paragraph. Auxiliary fields for Kähler moduli correspond locally on the target space to intrinsic torsion classes for background with $S U(3) \times S U(3)$ structure. However, when the two auxiliary fields in a given multiplet are dialed independently of each other, the string background is generically nongeometric. ${ }^{-1}$ This need for nongeometric structures becomes clear from the worldsheet, as we will discuss. Furthermore, one can understand why mirror symmetry may still be valid from the worldsheet point of view: it corresponds to reversing the sign of a $U(1)_{R}$ current, which exists even though it is no longer conserved.

Note that while the torsion classes are typically defined without reference to an underlying (pre-deformation) Calabi-Yau manifold, the picture that we adopt here is that one starts with an ordinary Calabi-Yau compactification, and then deforms that compactification as parametrized by the fluxes and torsion. However, it is not known that good compact examples of the types we discuss are related by any physical process (such as domain walls [5,37]) or sensible mathematical deformation to a Calabi-Yau background with D-branes and orientifolds. For noncompact local models, however, one can make

${ }^{2}$ Here, purely electric NS-NS flux means flux through the $A$ cycles but not $B$ cycles, in a symplectic basis of $\mathrm{H}_{3}$.

3 Here, $S U(3) \times S U(3)$ refers to the structure group of an extension of the bundle $T \oplus T^{*}$, or equivalently, to distinct left and right moving $S U(3)$ structures of the usual frame bundle.

4 Note that Refs. [19,20,21] also consider backgrounds with " $R$-flux," which are not even locally geometric. We suspect that we are missing the fluxes because we are considering deformations of a geometric background. 
arbitrarily small, continous deformations of the flux and torsion classes, and we will to a large degree focus on such models here. (Our observations about the relationship to nongeometric models also holds for compact examples, but the specific arguments there do not depend on any relation to a nearby Calabi-Yau). At any rate, we hope that the current work, combined with the many results regarding the action of mirror symmetry on D-branes, will be a useful guide to the story for fully compact models.

The fact that the compactifications we study are globally nongeometric demonstrates that supergravity is insufficient. Furthermore, the auxiliary fields for hypermultiplets in type IIB induce superpotentials for the Kähler moduli of the underlying Calabi-Yau geometry; at the minimum of the resulting potential, the volumes of some cycles will generically be string scale. In $\S 6$, we provide direct arguments that worldsheet instanton effects are important. The reader may sensibly object that most of our analysis nonetheless uses the supergravity approximation. To the extent that we study local noncompact models, and can consider the hypermultiplet auxiliary fields to parametrize small, continuous

deformations of Calabi-Yau backgrounds (as opposed to discrete deformations), we are on good footing. Compact models will require a stringy version of the mathematics of $S U(3) \times S U(3)$ structure, as well as need to satisfy additional constraints, as in [38]. Hopefully, the worldsheet perspective in this paper will provide the first step in this direction. More generally, we feel that our worldsheet perspective gives a useful organization of and insight into generalized geometries.

\section{Review}

In this section, we review some facts about compactifications that give $\mathcal{N}=2$ effective actions; this means that the Lagrangian is invariant under off-shell $\mathcal{N}=2$ supersymmetry transformations, but expectation values for auxiliary fields break the $\mathcal{N}=2$ supersymmetry. We open in $\S 2.1$ with a discussion of the $\mathcal{N}=2$ superspace expansion of 39-42 for hypermultiplets, and discuss the auxiliary field structure. In $\S 2.2$ we review the relationship between spacetime supersymmetry in four dimensions and $G$-structures on the compactification manifolds. In $\S 2.3$ we review the relationship between spacetime and worldsheet supersymmetry. Finally, in $\S 2.4$ and $\S 2.5$ we review some basic facts about $S U(3)$ structure and $S U(3) \times S U(3)$ structure, respectively. 


\section{1. $\mathcal{N}=2$ superspace expansion of hypermultiplets}

In this paper, we study type IIB theories with closed string modes that lie in $\mathcal{N}=2$ supermultiplets. As described in the previous section, this makes sense in models for which $\mathcal{N}=2$ supersymmetry is broken to $\mathcal{N}=1$ at a lower scale than the compactification scale, or is so broken by local defects in the compactification. Many of the type II flux compactification models that have dominated the recent literature on string model-building fall into this class [43,44], as do Hořava-Witten compactifications [45,46] for a broad range of parameters consistent with coupling constant unification [47,48]. We focus on type IIB models in this paper.

Following Ref. [3], our interest is in breaking $\mathcal{N}=2$ supersymmetry to $\mathcal{N}=1$ or $\mathcal{N}=0$ through expectation values of bosonic auxiliary fields in $\mathcal{N}=2$ multiplets. These expectation values appear in nonzero (non total derivative) supersymmetry transformations of the fermions, so that the state breaks supersymmetry. Note that consistent $\mathcal{N}=1$ or $\mathcal{N}=0$ compactifications to four dimensions include orientifolds which may project out half or all of an $\mathcal{N}=2$ multiplet. However, if $\mathcal{N}=1$ supersymmetry survives to low energies, the surviving closed string fields should descend from the original $\mathcal{N}=2$ theory via the orientifold projection, and the surviving auxiliary fields in the $\mathcal{N}=1$ multiplets control the SUSY-breaking terms of this more realistic model.

In [1,2], the focus was on vector multiplets of the underlying type IIB $d=4, \mathcal{N}=2$ model, and on hypermultiplets of type IIA. In this work we focus on the hypermultiplets of type IIB (our results will also give the NS-NS auxiliary fields for the type IIA vector multiplets). There are various off-shell extensions of $\mathcal{N}=2$ multiplets whose on-shell bosons all have spin zero. However, there is a particular off-shell extension that appears to be natural from the point of view of the string worldsheet. It follows from the $\mathcal{N}=2$ superspace formalism of Refs. [39, 40,41,42]. In this formalism, the anticommuting superspace coordinates are a pair of spinor-valued Grassmann variables $\left(\theta_{\alpha}, \hat{\theta}_{\alpha}\right)$ and their complex conjugates $\left(\bar{\theta}_{\dot{\alpha}}, \hat{\bar{\theta}}_{\dot{\alpha}}\right)$. Each pair is a doublet of Weyl spinors under the $S U(2)_{R}$ symmetry of $d=4, \mathcal{N}=2$ supersymmetry. If we choose a direction in the doublet representation of $S U(2)_{R}$, the corresponding Weyl spinor is the superspace Grassmann variable of the $\mathcal{N}=1$ subalgebra of the $\mathcal{N}=2$ supersymmetry.

The doublet of Grassmann variables arises very naturally from the worldsheet [1,2, 2, 2]. For type II strings with $\mathcal{N}=(2,2)$ worldsheet supersymmetry, currents for spacetime supersymmetry can be constructed from both the left- and right-moving sectors of the 
worldsheet 49,50, leading to a natural decomposition of $\mathcal{N}=2$ spacetime supersymmetry into two $\mathcal{N}=1$ subalgebras. The spacetime supersymmetries formed from the left- and right-moving sectors of the worldsheet form an $S U(2)_{R}$ doublet; the $S U(2)_{R}$ symmetry is nonperturbative on the worldsheet. As in [42], we take $\theta$ to be the superspace variable corresponding to the $\mathcal{N}=1$ subgroup of the spacetime supersymmetry built from the left-moving sector of the worldsheet, and $\hat{\theta}$ to be the superspace variable corresponding to the $\mathcal{N}=1$ subgroup of the spacetime supersymmetry built from right-moving worldsheet fields.

The $\mathcal{N}=2$ superfield for a hypermultiplet is chiral with respect to the left moving supersymmetry and anti-chiral with respect to the right-moving supersymmetry. Its expansion in the superspace Grassman variables is:

$$
\begin{aligned}
\mathcal{H}^{a}= & w^{a}+\theta^{\alpha} \chi_{\alpha}^{a}+\hat{\bar{\theta}}^{\dot{\beta}} \hat{\bar{\chi}}_{\dot{\beta}}^{a}+\theta^{2} y^{a}+\hat{\bar{\theta}}^{2} \hat{\bar{y}}^{a} \\
& +\theta^{\alpha} \hat{\bar{\theta}}^{\dot{\beta}} \sigma_{\alpha \dot{\beta}}^{\mu} F_{\mu}^{a}+\theta^{\alpha} \hat{\bar{\theta}}^{2} \eta_{\alpha}^{a}+\hat{\bar{\theta}}^{\dot{\beta}} \theta^{2} \hat{\bar{\eta}}_{\dot{\beta}}^{a}+\theta^{2} \hat{\bar{\theta}}^{2} C^{a} .
\end{aligned}
$$

Here $w^{a}$ is a complex scalar, and $F_{\mu}=\partial_{\mu} \varphi^{a}$, where $\varphi^{a}$ is also a complex scalar; $y^{a}, \hat{\bar{y}}^{a}$ and $C^{a}$ are auxiliary fields, and $a$ simply labels the moduli and runs over the appropriate range of values, i.e.,

$$
\begin{array}{ll}
a=1, \ldots, h^{(1,1)} & \text { type IIB, } \\
a=1, \ldots, h^{(1,2)} & \text { type IIA, }
\end{array}
$$

in a Calabi-Yau compactification.

\section{Hypermultiplets in type IIB}

Consider type IIB string theory compactified on a Calabi-Yau manifold $X$, with $\left\{\omega_{a}\right\}$ a basis of $H^{(1,1)}(X)$. The Kähler form can be written as $J=t^{a} \omega_{a}$, where the $t^{a}$ are the real Kahler moduli of the compactification. Vacuum expectation values $B=b^{a} \omega_{a}$ for the NS-NS 2-form potential also preserve spacetime supersymmetry. In this case we define complexified Kähler moduli $w^{a}=b^{a}+i t^{a}$, which are again purely NS-NS fields. Supersymmetry transformations act with a half unit of spectral flow, mapping the NS sector to the R sector and vice-versa. Therefore, the field, $\varphi^{a}$ in Eq. (2.1) is an RR field, and $y^{a}$ is an NS-NS auxiliary field. The field $\varphi^{a}$ can be constructed as follows. The RR two-form $C^{(2)}$ contributes massless modes via the decomposition $C=\sum_{a} c^{a} \omega_{a}$. The RR

four-form $C^{(4)}$ gives four-dimensional two-form potentials dual to scalars, via the expansion $C^{(4)}=\sum_{a} \tilde{c}_{\mu \nu}^{a} \omega_{a}$. We write $\partial_{\mu} \varphi^{a}=\partial_{\mu} c^{a}+i\left({ }^{*} d \tilde{c}^{a}\right)_{\mu}$. 
The universal hypermultiplet, which includes the four-dimensional dilaton $\phi$ (not to be confused with the RR scalars $\varphi^{a}$ just discussed), arises in a different way. For this multiplet, the natural complex NS-NS scalar is written $w^{\phi}=a+i e^{-2 \phi}$, where the pseudoscalar $a$ is the four-dimensional dual of the NS-NS 2-form $B_{\mu \nu}$. However, the worldsheet naturally couples to $B_{\mu \nu}$, not $a$. Correspondingly, in Ref. [42], the dilaton has a different superfield description than that described in Eq. (2.1): one decomposes a real scalar $\mathcal{N}=2$ superfield into superfields for the graviton and dilaton multiplets, and the NS-NS 2-form arises directly in the latter. The corresponding auxiliary fields are Ramond-Ramond. However, from a four- or ten-dimensional spacetime point of view, there is no problem in principle with writing the dilaton multiplet in the form (2.1) with $w^{\phi}$ as described, even if there is no obvious vertex operator description of $w^{\phi}$. We will find a thus natural candidate for $y^{\phi}$ from a spacetime rather than worldsheet point of view. It would be interesting and important to construct the auxiliary fields in the dilaton multiplet as presented in [42]. It may also be important to understand the auxiliary fields in other off-shell presentations of the hypermultiplets, such as the various multiplets that arise from projective superspace [51]. In particular, some string compactifications in the literature - e.g. [52,53] - have F-terms in the dilaton hypermultiplets which are combinations of NS-NS and R-R fields, as we will discuss below.

In [42, the number of off-shell degrees of freedom in the multiplet (2.1) is reduced by imposing additional reality conditions $\partial^{2} \mathcal{H}=\hat{\partial}^{2} \mathcal{H}=0$, where $\partial, \hat{\partial}$ are defined in [42]. We find that these constrain the components of $\mathcal{H}$ such that $C^{*} \propto \partial^{2} w$, and $\hat{\bar{y}}=y^{*}$ (which is slightly different from the condition written in [42]). In the discussion below, we will not impose such conditions, so that $y$ and $\hat{\bar{y}}$ are independent. This is consistent with the type IIA picture discussed in [1]. For example, a background with $y=0$ and $\hat{\bar{y}} \neq 0$ corresponds to a background with an $\mathcal{N}=1$ supersymmetry preserved (with Grassmann superspace variables $\theta$ ). A noncompact example is the solution of [54,55], as discussed in [1].

\subsection{Spacetime supersymmetry and G-structures}

In ten dimensions, type IIB string theory contains two supercharges $Q_{N}$ of the same chirality. The supersymmetry transformations are parametrized by two ten-dimensional positive-chirality Majorana-Weyl spinors $\epsilon_{N}$, where $N=1,2$. For compactifications to four dimensions, we write (cf. Ref. [10])

$$
\epsilon_{N}=\zeta_{N+} \otimes \eta_{-}^{N}+\zeta_{N-} \otimes \eta_{+}^{N}
$$


where $\zeta_{N \pm}$ are four dimensional spinors, $\eta_{ \pm}^{N}$ six dimensional spinors, and the subscripts \pm denote four dimensional and six dimensional chirality, respectively. 5

$\mathcal{N}=2$ supersymmetry and $S U(3) \times S U(3)$ structures.

We begin by considering a compactification which is locally a smooth six dimensional manifold $M$, which is well described by supergravity.6 Following [10,18, we demand that the full effective action (including all of the massive Kaluza-Klein and string modes) be invariant under $\mathcal{N}=2$ supersymmetry. Note that this condition is compatible with the presence of nonvanishing expectation values of auxiliary fields: the action is still invariant under $\mathcal{N}=2$ supersymmetry, but the state with these expectation values is not. Expectation values for the auxiliary fields break $\mathcal{N}=2$ to $\mathcal{N}=1$ or $\mathcal{N}=0$.

For the action of $\mathcal{N}=2$ supersymmetry to be well-defined, the spinors $\eta^{N}$ must be globally well-defined. When the solution is smooth and reliably described by supergravity, one typically demands, as in [10, 18], that the spinors are also nowhere-vanishing. This condition usually follows from the demand that for a supersymmetric background, the spinor $\eta^{N}$ be covariantly constant, which implies that its norm is constant.

When the supersymmetry is nonlinearly realized, and still described by a nowherevanishing spinor, it is possible for the corresponding $\mathcal{N}=2$ supersymmetry to be broken at a low scale compared to the Kaluza-Klein scale. Spinors that do vanish at points or at loci of finite codimension cannot be covariantly constant, and in nonsingular geometric compactifications correspond to a broken supersymmetry in the set of local ten-dimensional supersymmetries. We expect the energy scale of breaking to generically be the KaluzaKlein scale. 6

In the work described here, we have in mind the case that the $\mathcal{N}=2$ supersymmetry is broken by expectation values of auxiliary fields at a low scale compared to the Kaluza-Klein scale. We therefore consider backgrounds with two nowhere-vanishing spinors. For a spinor to be globally well-defined, it must be invariant under the structure group $G \subset \operatorname{Spin}(6)$

5 The assigment of $6 \mathrm{~d}$ chirality follows from the definition of the four-dimensional chirality operator as $\gamma^{5}=i \gamma^{0} \ldots \gamma^{3}$ and the $6 \mathrm{~d}$ chirality operator as in the Appendix. Using the definitions of the 10d Clifford algebra in eq. (2.2) of [10], then $\Gamma^{11}=\Gamma^{0} \ldots \Gamma^{9}=-\gamma_{(4 d)}^{5} \otimes \gamma_{(6 d)}^{7}$.

6 However, we will point out in $\S 3, \S 6$ that the vacua in truly compact $S U(3) \times S U(3)$ structure models will generically have string-scale features; this is one of many dangerous games we play in this paper.

7 We thank D. Waldram and especially M. Graña for patient correspondence on these points. 
of the spinor bundle on $M$. Thus, the decomposition of the 4 of Spin(6) into irreducible representations of $G$ must contain a singlet. The generic (i.e. largest) structure group $G$ with these properties is $S U(3)$. Each invariant, nowhere-vanishing spinor $\eta^{N}$ defines an $S U(3)$ structure on the spinor bundle. (This structure group is inherited by the frame bundle 8 on $M$, so it is also possible to describe an $S U(3)$ structure in terms of the frame bundle without reference to spinors.)

Since the $\operatorname{Spin}(6)$ spinors $\eta_{ \pm}^{1}$ and $\eta_{ \pm}^{2}$ of Eq. (2.3) need not be proportional to one another, each generically defines an $S U(3)$ structure distinct from the other. Thus, we have two structures, $S U(3)_{N}$, for $N=1,2$. This is natural from the point of view of the worldsheet, as we discuss further below: the spinors $\eta^{1}$ and $\eta^{2}$ are generated from the left- and right-moving sectors of the worldsheet, respectively. Each chiral sector of the worldsheet has its own associated $S U(3)$ structure.

It is possible to combine the two $S U(3)_{N}$ structures into a single $S U(3) \times S U(3)$ structure in the "generalized complex geometry" of Hitchin et al [10,56,57]. In this case, the generalized tangent space of interest is (a bundle extention of) $T \oplus T^{*}(M)$, with structure group $S U(3) \times S U(3)$. In this generalized geometry, the role played by the $S U(3)_{N}$ invariant Spin(6) spinors $\eta_{ \pm}^{N}$ in the previous discussion is now played by $S U(3)_{1} \times S U(3)_{2}$ invariant $\operatorname{Spin}(6,6)$ pure spinors: $\Omega_{+} \propto \operatorname{Re} \eta_{+}^{1} \otimes \eta_{+}^{2}$ of positive chirality and $\Omega_{-} \propto \operatorname{Re} \eta_{+}^{1} \otimes \eta_{-}^{2}$ of negative chirality. In this way of writing the pure spinors, $S U(3)_{1}$ acts on the left and $S U(3)_{2}$ acts on the right. To reproduce the earlier discussion, all that is needed is a projection from $T \oplus T^{*}(M)$ to $T(M)$. As described in Ref. [57], there are two canonical choices of this projection. One gives the group $S U(3)_{1}$ associated to $\eta_{ \pm}^{1}$, and the other gives the group $S U(3)_{2}$ associated to $\eta_{ \pm}^{2}$.

These various ways of encoding $S U(3) \times S U(3)$ structure are closely related to the various presentations of the "doubled torus" in [58] used to describe stringy torus fibrations. (See, for example, [59] for a systematic discussion of this formalism.) In that work, one replaces a $T^{n}$ factor (or fiber) in the target space with $T^{2 n}$, on which the T-duality group acts linearly. One may choose a polarization that splits this torus into two $n$-dimensional factors. One choice is to split them into two $T^{n}$ factors described by left- or right-moving

8 The frame bundle is defined by its sections: a local section of the frame bundle is a choice of vielbein basis, i.e., a "frame" of six 1-forms (or vectors) on each open set $U \in M$. Usually, there is no global section; in the special case that one exists, there are six global 1-forms $e^{A}$, and $M$ is said to be parallizable. 
chiral bosons on the worldsheet. This is analogous to the tack we will take in this paper. Alternatively, one may split the doubled torus into a direct sum of the original torus and its dual. This is closer in spirit to the discussion in [56,57].

$\mathcal{N}=4$ supersymmetry and local versus global $S U(2)$ structures

For a generic $S U(3) \times S U(3)$ structure, the spinors are locally independent, and are only parallel at isolated points. If they are never parallel, then the two spinors define an $S U(2)$ structure. This allows one to define an $\mathcal{N}=4$ supersymmetry acting on the four-dimensional theory, by reducing each of the ten-dimensional spinors $\epsilon_{1,2}$ on either of the six-dimensional spinors $\eta_{1,2}$.

If the spinors are parallel at points, there is a local but not a global $S U(2)$ structure. In principle, one could still reduce each of $\epsilon_{1,2}$ on either of $\eta_{1,2}$ and so define an $\mathcal{N}=4$ supersymmetry. However, the fact that the spinors $\eta_{1,2}$ coincide at points means that one of the putative supercharges in the $\mathcal{N}=4$ algebra will come from a reduction on a spinor which vanishes at specific points in the moduli space. As discussed above, this means that the $\mathcal{N}=4$ supersymmetry will be broken to $N \leq 2$, generically at the Kaluza-Klein scale.

\subsection{Worldsheet vs. spacetime supersymmetry}

$\mathcal{N}=2$ spacetime supersymmetry in four dimensions requires $\mathcal{N}=(2,2)$ worldsheet supersymmetry for the $c=9$ superconformal field theory describing the compact CFT [49,50]. The $S U(2)_{R}$ doublet of supercharges in the $(-1 / 2,-1 / 2)$ picture can be written as:

$$
\left(\begin{array}{c}
Q_{L, \alpha}(z) \\
\hat{Q}_{R, \beta}(\bar{z})
\end{array}\right)=\left(\begin{array}{c}
e^{-\phi_{L} / 2} S_{\alpha, L} \bar{\Sigma}_{L}(z) \\
e^{-\phi_{R} / 2} S_{\beta, R} \bar{\Sigma}_{R}(\bar{z})
\end{array}\right)
$$

Here $\phi_{L, R}$ come from the bosonization of the superconformal ghosts, $S_{\alpha}$ are the spin fields

for the $\mathbb{R}^{4}$ sigma model factor of the CFT, and $\bar{\Sigma}_{L, R}$ are the $U(1)$ charge $-\frac{3}{2}$ spectral flow operators for the $c=9$ compact SCFT, mapping NS $\leftrightarrow \mathrm{R}$. If the compact CFT is a sigma model, then $\Sigma_{L, R}$ can be written as spin fields for the sigma model coordinates, and transform in the spinor representation of Spin(6). Supersymmetry requires that this be a singlet of $S U(3) \subset S O(6)$. Thus there is a map between the spectral flow operators and these spinors.

The standard example of a sigma model with $\mathcal{N}=(2,2)$ worldsheet supersymmetry is one with a Calabi-Yau target space $M$. In this case $\eta_{ \pm}^{1}=\eta_{ \pm}^{2}=\eta_{ \pm}$and $\nabla_{m} \eta_{ \pm}=0$. The Levi-Civita connection on $M$ has $S U(3)$ holonomy, and thus $M$ is guaranteed to be 
Ricci-flat and Kähler. This is not the most general possibility for $\mathcal{N}=(2,2)$ sigma models [60]. In the presence of nonvanishing NS-NS three-form flux, $\mathcal{N}=(2,2)$ supersymmetry is preserved if there are two almost complex structures $J_{ \pm}$such that

$$
\nabla_{\mu} J_{ \pm \lambda}^{\nu} \mp \frac{1}{2}\left(H_{\mu \rho}^{\nu} J_{ \pm \lambda}^{\rho}-H_{\mu \lambda}^{\rho} J_{ \pm \rho}^{\nu}\right)=0 .
$$

Here, $J_{+}$and $J_{-}$should be identified with $J^{1}$ and $J^{2}$ of Sec. 2 . If $\psi_{L}^{\mu}(z), \psi_{R}^{\mu}(\bar{z})$ are the leftand right-moving spacetime fermions polarized along $M$, then $J_{L, R}=J_{L, R, \mu \nu} \psi_{L, R}^{\mu} \psi_{L, R}^{\nu}$ are the left and right moving worldsheet $U(1)_{R}$ currents in the $\mathcal{N}=(2,2)$ algebra. One may construct $\Sigma_{L, R}$ by bosonizing these $U(1)$ currents, and these will be mapped to spinors which satisfy

$$
\nabla_{\mu} \eta \pm \frac{1}{8} H_{\mu \nu \rho} \Gamma^{\nu \rho} \eta=0,
$$

where the \pm is correlated with the $d=10, \mathcal{N}=2$ supersymmetry from which $\eta^{N}$ descends (i.e., $+\leftrightarrow \eta^{1}$ and $-\leftrightarrow \eta^{2}$ ). These backgrounds correspond to a particular class of $S U(3) \times S U(3)$ structure compactifications. We are working with an off-shell presentation of the hypermultiplets (2.1) for which the auxiliary fields are NS-NS. Other presentations may involve RR fields. The RR flux would modify (2.5) and (2.6), and may break additional supersymmetry. However, since we do not know how to treat RR backgrounds in the RNS worldsheet formalism, we will not study these effects in this section (see 61]).

We will be particularly interested in cases where $\mathcal{N}=2$ spacetime supersymmetry is broken. If the supersymmetry is broken by NS-NS deformations to $\mathcal{N}=1$ and the dilaton does not become too large, the worldsheet supersymmetry is generically broken to $\mathcal{N}=$ $(2,1)$; if spacetime supersymmetry is broken entirely then the worldsheet supersymmetry is broken to $\mathcal{N}=(1,1)$. It is also possible that supersymmetry is broken simply because the physical states no longer satisfy the $R$-charge quantization rule described in [49,50]. We believe that in terms of the $\mathcal{N}=1$ spacetime supersymmetry associated with this $R$-symmetry, this breaking will be through D-terms, as is true in the open string case [62]. Following that work, we expect the argument to run like this: the complex NS-NS scalars are described by a vertex operator with $U(1)_{R}$ charge, and so a change of the R-charge affects equally both real scalars in the spacetime multiplet. In particular the mass shifts will be the same for both. This is characteristic of D-term breaking; F-term breaking leads to mass splittings between scalars in chiral multiplets. The D-terms are auxiliary fields in the vector multiplets. We leave verification of this scenario for future work. Meanwhile, the statement that auxiliary fields in hypermultiplets break some of the $\mathcal{N}=(2,2)$ worldsheet 
supersymmetry is fully consistent with the results in this paper. In particular this typically means that one or both of the $U(1)_{R}$ symmetries are broken.

The worldsheet manifestation of this is as follows. When spacetime supersymmetry is broken through expectation values for the auxiliary fields $y, \hat{\bar{y}}$, the $\mathcal{N}=2$ transformations will still act on the fields, albeit nonlinearly. On the worldsheet, we will find that the operators corresponding to $y, \hat{\bar{y}}$ explicitly break the $U(1)_{R}$ charge. The $R$-current exists but no longer satisfies (2.5); as we will describe below, the left hand side will contain torsion terms.

\subsection{Review of $S U(3)$ structure}

The most general case that we are interested in has two $S U(3)$ structures. To understand them it is helpful to focus first on one $S U(3)$ structure - this will also describe the well-studied case in which the two $S U(3)$ structures are parallel and can be made identical.

Manifolds with $S U(3)$ structure can be classified by a set of intrinsic torsion classes. These encode the failure of the corresponding positive and negative chirality spinors $\eta_{ \pm}$to be covariantly constant with respect to the Levi-Civita connection:

$$
\nabla_{m} \eta_{ \pm}=\left(q_{m}+i \tilde{q}_{m} \gamma_{7}\right) \eta_{ \pm}+i q_{m n} \gamma^{n} \eta_{\mp}
$$

where $\gamma_{7}$ is the six-dimensional chirality operator, and $\tilde{q}_{m}, q_{m}, q_{m n}$ are determined by the intrinsic torsion of the manifold [11].

Alternatively, one may define an almost complex structure via:9

$$
J_{m n}=-i \bar{\eta}_{ \pm} \gamma_{m n} \gamma_{7} \eta_{ \pm}
$$

where $\eta_{ \pm}^{\dagger} \eta_{ \pm}=1.10$ The torsion classes measure the failure of $J$ to be covariantly conserved. A third description [8] is as follows: Define the two-form $J$ with coefficients $J_{m n}$; this has index structure $(1,1)$ with respect to the almost complex structure. Define also the $(3,0)$ form $\Omega$ with coefficients

$$
\Omega_{m n p}=-i \bar{\eta}_{-} \gamma_{m n p} \eta_{+}
$$

\footnotetext{
9 See Appendix A for a complete discussion of our normalization conventions.

10 Note that our definitions and normalizations differ by factors of 2 from those given in [10, 18]. Our definitions are consistent with the conventions given in Appendix A. In particular there is a factor of 2 difference that appears in the Fierz identity given in Appendix A.
} 
The torsion classes, which measure the deviation of the $S U(3)$ structure manifold from having (Levi-Civita) $S U(3)$ holonomy, can then be defined as:

$$
\begin{aligned}
& d J=-\frac{3}{2} \operatorname{Im}\left(W_{1} \bar{\Omega}\right)+W_{4} \wedge J+W_{3} \\
& d \Omega=W_{1} J^{2}+W_{2} \wedge J+\bar{W}_{5} \wedge \Omega .
\end{aligned}
$$

Here $W_{1}$ is a complex 0 -form, $W_{2}$ is a complex $(1,1)$ form where $W_{2} \wedge J$ is primitive with respect to $J_{m n}, W_{3}$ is a real primitive $(2,1) \oplus(1,2)$ form, $11 W_{4}$ is a real one-form, and $W_{5}$ is a $(1,0)$ form. Note also that $d J$ can include a $(3,0) \oplus(0,3)$ piece in addition to a $(1,2) \oplus(2,1)$ component because the almost complex structure is generically not integrable. Similarly, $d \Omega$ can include $(2,2)$ components. Using the Fierz identities given in Appendix A, we can define $q_{m}, \tilde{q}_{m}$ and $q_{m n}$ in terms of the $W_{i}$.

Each of the $W_{i}$ lives in a definite representation of the $S U(3)$ structure group. Any given representation is most easily found by noting that holomorphic indices (with respect to the almost complex structure $J_{m}{ }^{n}$ ) lie in the $\mathbf{3}$ of $S U(3)$, while antiholomorphic indices lie in the $\overline{\mathbf{3}}$ of $S U(3)$. Thus, $W_{1}$ is a complex $S U(3)$ singlet; $W_{2}$ is a complex form in the $\mathbf{8}$ of $S U(3) ; W_{3}$ in the $\mathbf{6} \oplus \overline{\mathbf{6}}$ of $S U(3)$; and $W_{4}, W_{5}$ lie in the $\mathbf{3} \oplus \overline{\mathbf{3}}$ of $S U(3)$.

Following [11, 12, 13, 15], we can similarly expand the 3 -form $H$ in this $(J, \Omega)$ basis as

$$
H=-\frac{3}{2} \operatorname{Im}\left(H_{1} \bar{\Omega}\right)+H_{3}+H_{4} \wedge J
$$

and we will find it useful to do so in the following sections. The $H_{k}$ lie in the same representations as $W_{k}$, for $k=1,3,4$.

\section{Intrinsic torsion and the spin connection}

Our computations in $\S 3$ will use the relationship between the intrinsic torsion and the components of the spin connection decomposed according to the almost complex structure.

Given a vielbein $\left\{e^{A}\right\}, A=1, \ldots, 6$, considered as a collection of one-forms, we define a complex vielbein

$$
\begin{aligned}
& e^{a}=e^{A=2 a-1}+i e^{A=2 a} \\
& e^{\bar{a}}=e^{A=2 a-1}-i e^{A=2 a},
\end{aligned}
$$

where $a=1,2,3$. In this basis [8]

$$
\begin{aligned}
& J=i g_{a \bar{a}} e^{a} \wedge e^{\bar{a}} \\
& \Omega=e^{1} \wedge e^{2} \wedge e^{3},
\end{aligned}
$$

11 A form $\omega$ is primitive with respect to $J$ if $\omega \wedge J=0$. 
where $g_{a \bar{a}}=\frac{1}{2} \eta_{a \bar{a}}=\frac{1}{2} \delta_{a \bar{a}}$ is the flat metric in complex coordinates. The 2 -form $J$ defines an almost complex structure after raising one index with the inverse metric $g^{\bar{a} a}$.

Using the Cartan structure equations,

$$
\begin{aligned}
d e^{a} & =-\omega^{a}{ }_{c} \wedge e^{c}-\omega^{a}{ }_{\bar{c}} \wedge e^{\bar{c}} \\
& =-\omega_{b}{ }^{a}{ }_{c} e^{b} \wedge e^{c}-\omega_{\bar{b}}{ }^{a}{ }_{c} e^{\bar{b}} \wedge e^{c}-\omega_{b}{ }^{a}{ }_{\bar{c}} e^{b} \wedge e^{\bar{c}}-\omega_{\bar{b}}{ }^{a}{ }_{\bar{c}} e^{\bar{b}} \wedge e^{\bar{c}}
\end{aligned}
$$

combined with (2.10) and (2.13), we can relate components of the spin connection with specific complex indices to different intrinsic torsion classes. Specifically, we find that

$$
\begin{aligned}
W_{1} & =\frac{4}{3} \epsilon^{\bar{a} \bar{b} \bar{c}} \omega_{\bar{a} \bar{b} \bar{c}} ; \quad H_{1}=-\frac{2 i}{9} \epsilon^{\bar{a} \bar{b} \bar{c}} H_{\bar{a} \bar{b} \bar{c}} \\
\left(W_{2}\right)_{a \bar{b}} & =i \Omega^{\bar{c} \bar{d}}{ }_{a} \omega_{\bar{c} \bar{d} \bar{b}}-\frac{i}{3} g_{a \bar{b}} \Omega^{\bar{c} \bar{d} \bar{f}} \omega_{\bar{c} \bar{d} \bar{f}} .
\end{aligned}
$$

(See also [63].) Note in particular that the totally antisymmetric part of $\omega$ will not contribte to $W_{2}$.

\subsection{Review of $S U(3) \times S U(3)$ structure}

We will find that for general values of the hypermultiplet auxiliary fields $y$ and $\hat{\bar{y}}$, the $\operatorname{Spin}(6)$ spinors $\eta^{N}(N=1,2)$ are not parallel, so that we have two distinct $S U(3)$ structures. Locally on $M$, the two spinors are independent almost everywhere, and may become parallel only at isolated points. In a neighborhood in which the two spinors are everywhere independent, the intersection $S U(2)=S U(3)_{1} \cap S U(3)_{2}$ defines a local $S U(2)$ structure. However, this intersection is enlarged to $S U(3)$ at the special points where the two spinors $\eta^{N}$ become parallel. Therefore, globally, there are two $S U(3)$ structures, but there is no global $S U(2)$ structure.

Given the doublet $\eta^{N}$, one may define a doublet of real 2-forms $J^{N}$ and a doublet of complex three forms $\Omega^{N}$,

$$
J_{m n}^{N}=-\frac{i}{2} \bar{\eta}^{N} \gamma_{m n} \gamma_{7} \eta^{N} \quad \text { and } \quad \Omega_{m n p}^{N}=-\frac{i}{2} \bar{\eta}^{N} \gamma_{m n p}\left(1+\gamma_{7}\right) \eta^{N}
$$

Here, the pair $\left(J^{N}, \Omega^{N}\right)$ provides an equivalent definition of the $S U(3)_{N}$ structure. Accordingly we have a doublet $W_{1}^{N}, \ldots, W_{5}^{N}$ of the five torsion classes defined in Eq. (2.10). We will argue that $N=1,2$ correspond to $y, \hat{\bar{y}}$, respectively. Furthermore, for each $S U(3)$ structure, there is an almost complex structure with respect to which we can write a vielbein with complex tangent frame indices. For each such almost complex structure, the intrinsic torsion classes $W_{k}^{N}$ can be written in terms of the spin connection as in (2.15).

Instead of defining a doublet of $S U(3)_{N}$ torsion classes, one could instead define a single set of $S U(3) \times S U(3)$ torsion classes; typically, one would define them in terms of the pure spinors built from $\eta_{ \pm}^{N}$, as in [11]. However, the two descriptions are equivalent, and the formulation given here will be the most useful for our purposes. 


\section{Worldsheet vertex operators for auxiliary fields}

In this section we will follow [1,2] and use worldsheet techniques to compute $y, \hat{\bar{y}}$ for Kähler moduli in Calabi-Yau compactifications of type IIB string theory.

\subsection{Worldsheet supersymmetry and target space geometry}

Expectation values $y \neq 0$ or $\hat{\bar{y}} \neq 0$ break the left- or right-moving $\mathcal{N}=2$ superconformal symmetries, respectively, down to $\mathcal{N}=1$. In particular, $\hat{\bar{y}} \neq 0, y=0$, corresponds to a background with $\mathcal{N}=(2,1)$ supersymmetry. This observation will be useful in interpreting the vertex operator calculation.

A general $\mathcal{N}=(1,1)$ worldsheet sigma model with NS-NS $H$-flux has the fermion bilinear terms 12

$$
\begin{gathered}
\mathcal{L}=-i g_{\mu \nu} \psi_{+}^{\mu} \mathcal{D}_{-} \psi_{+}^{\nu}-i g_{\mu \nu} \psi_{-}^{\mu} \mathcal{D}_{+} \psi_{-}^{\nu} \\
g_{\mu \nu} \psi_{ \pm}^{\mu} \mathcal{D}_{\mp} \psi_{ \pm}^{\nu} \equiv g_{\mu \nu} \psi_{ \pm}^{\mu} \partial_{\mp} \psi_{ \pm}^{\nu}+\left(g_{\mu \nu} \Gamma_{\lambda \rho}^{\nu} \pm \frac{1}{2} H_{\mu \lambda \rho}\right) \psi_{ \pm}^{\mu} \partial_{\mp} X^{\lambda} \psi_{ \pm}^{\rho}
\end{gathered}
$$

where $\psi_{-}\left(\psi_{+}\right)$are left- (right)-moving worldsheet fermions. In addition, there are fourfermion terms of the form $R_{ \pm} \psi \psi \psi \psi$ where $R_{ \pm}$is curvature built from the connection $\Gamma_{ \pm, \nu \rho}^{\mu}=\Gamma_{\nu \rho}^{\mu} \mp \frac{1}{2} H_{\nu \rho}^{\mu}$. Worldsheet $\mathcal{N}=2$ supersymmetry for either the left or the right movers requires the existence of a complex structure $J_{-}$or $J_{+}$, respectively, which satisfies (2.5) [64]. (If both $J_{ \pm}$satisfy (2.5), then the theory has $\mathcal{N}=(2,2)$ supersymmetry). This condition implies that the metric must be hermitian, the NS-NS three-form must be of holomorphic type $(2,1)$ with respect to the almost complex structure $J_{+}$or $J_{-}$, and

$$
H_{i j \bar{k}}= \pm T_{i j \bar{k}} \equiv \pm i\left(\partial_{j} g_{i \bar{k}}-\partial_{i} g_{j \bar{k}}\right)
$$

where the sign in (3.2) is correlated with the sign \pm in $J_{ \pm}$. (In the case of $(2,2)$ supersymmetry, the geometry is bihermitian.)

\subsection{Vertex operators for auxiliary fields}

Vertex operators for auxiliary fields $y, \hat{\bar{y}}$ are calculated as follows [1, 2, 65, 66]. For $y^{a}$, we begin with the vertex operator for complexified Kähler modulus $w^{a}=b^{a}+i t^{a}$ in the $(-1,0)$ picture,

$$
V_{w^{a}}^{(-1,0)}=e^{-\phi_{-}(z)} \mathcal{O}_{\frac{1}{2}, 1}
$$

12 See Appendix A for our conventions for $H$. 
where $\mathcal{O}_{\frac{1}{2}, 1}$ has left-moving conformal dimension $\Delta=\frac{1}{2}$ and right-moving conformal dimension $\Delta=1$. Let $\Omega$ be the operator generating one unit of spectral flow (from NS to NS) on the left-movers: for Calabi-Yau compactifications, it is $\Omega=\Omega_{i j k} \psi_{-}^{i} \psi_{-}^{j} \psi_{-}^{k}$. The vertex operator for $y^{a}$ is found via the OPE:

$$
\Omega(z) \mathcal{O}_{\frac{1}{2}, 1}(u) \sim \frac{V_{y^{a}}}{(z-u)}+\text { nonsingular. }
$$

The vertex operator for $\hat{\bar{y}}$ is constructed identically by exchanging left and right movers. We are playing another dangerous game in writing such vertex operators, as they generally do not correspond to propagating modes. Nonetheless, following 65, 66, they do appear as coefficients of contact terms in certain OPEs, in precisely the fashion dictated by supersymmetry 13 Furthermore, the identification of these fields with specific fluxes and torsion classes on the manifold $M$ matches the spacetime arguments we will provide in $\S 4$ and $\S 5$.

Kähler moduli $w^{a}$ correspond to $(1,1)$ forms $\delta g_{i \bar{\jmath}}^{a}$. The $(-1,0)$ picture vertex operator is:

$$
V_{w^{a}}^{(-1,0)}=e^{-\phi_{-}} \delta g_{\bar{i} l}^{a} \psi_{-}^{\bar{i}} \partial_{+} X^{l}+e^{-\phi} \delta g_{i \bar{l}}^{a} \psi_{-}^{i} \partial_{+} X^{\bar{l}}
$$

In order to compute the OPEs, we will expand around a constant background field $X$ in Riemannian normal coordinates, and pretend that we are working at large radius.14 The scalars and fermions in this expansion have canonical kinetic terms, making a perturbative calculation of the OPEs straightforward. Let the vielbein be $e_{\mu}^{m}$, with $m=1, \ldots 6$ the frame indices, which can be organized into holomorphic and antiholomorphic indices $a, \bar{a}=1, \ldots 3$ by the almost complex structure, as discussed in $\S 2.4$ above. Following the discussion and notation of [68], we denote the components of the bosonic fluctuation relative to the vielbein basis by $\xi^{m}$, with fermionic superpartners $\psi^{m}=e_{\mu}^{n} \psi^{\mu}$. The quadratic fermion kinetic terms (3.1) become

$$
\begin{gathered}
\mathcal{L}=-i \eta_{m n} \psi_{+}^{m} D_{-} \psi_{+}^{n}-i \eta_{m n} \psi_{-}^{m} D_{+} \psi_{-}^{n} \\
\eta_{m n} \psi_{ \pm}^{m} D_{\mp} \psi_{ \pm}^{n} \equiv \psi_{ \pm}^{m}\left[\eta_{m n} \partial_{\mp} \psi_{ \pm}^{n}+\left(\omega_{m n p} \pm \frac{1}{2} H_{m n p}\right) \psi_{ \pm}^{n} \partial_{\mp} \xi^{p}\right]
\end{gathered}
$$

where $\omega_{m}^{n}{ }_{p}=e_{m}^{\mu} \omega_{\mu}^{n}{ }_{p}$ is the spin connection on $M$ with the 1-form index converted to the vielbein basis.

13 See [67 for a recent discussion of this in the context of open string theory.

14 As noted above, this is very dangerous. 
Let $\delta g_{i \bar{j}}$ be a deformation of the Kähler structure of the metric, corresponding to the scalar $w$ in a hypermultiplet. The corresponding $(-1,0)$-picture vertex operator is

$$
V_{w}^{(-1,0)}=e^{-\phi} \delta g_{\bar{a} b} \psi_{-}^{\bar{a}} \partial_{+} \xi^{b}+e^{-\phi} \delta g_{a \bar{b}} \psi_{-}^{a} \partial_{+} \xi^{\bar{b}},
$$

where $\delta g_{a \bar{b}} \equiv \delta g_{i \bar{\jmath}} e_{a}^{i} e_{b}^{\bar{\jmath}}$. Here and below, we have suppressed the upper index $a$ on $w$ and $\delta g$ to avoid confusion with the complex vielbein indices $a, \bar{a}$.

The spectral flow operator is:

$$
\Omega(z) \equiv \frac{1}{3 !} \Omega_{i j k} \psi_{-}^{i} \psi_{-}^{j} \psi_{-}^{k}=\frac{1}{6} \epsilon_{a b c} \psi_{-}^{a} \psi_{-}^{b} \psi_{-}^{c} .
$$

Using the operator product expansions $\psi_{-}^{\bar{a}} \psi_{-}^{b} \sim \eta^{\bar{a} b}$ and $\psi_{-}^{a} \psi_{-}^{b} \sim 0$, we find that

$$
V_{y}^{(0,0)}=\delta g_{\bar{a} c} g^{\bar{a} d} \Omega_{d a b} \psi_{-}^{a} \psi_{-}^{b} \partial_{+} \xi^{c},
$$

and similarly,

$$
V_{\hat{\bar{y}}}^{(0,0)}=\delta g_{a \bar{c}} g^{a \bar{d}} \bar{\Omega}_{\bar{d} \bar{a} \bar{b}} \psi_{+}^{\bar{a}} \psi_{+}^{\bar{b}} \partial_{-} \xi^{\bar{c}},
$$

with similar expressions when $i \delta g_{i \bar{\jmath}}$ is replaced by $\delta(B+i g)_{i \bar{\jmath}}$. We interpret these vertex operators as deformations of the Lagrangian (3.6). Due to the appearance of $\Omega_{d a b}$ and $\bar{\Omega}_{\bar{d} \bar{a} \bar{b}}$ in the expressions for $V_{y}$ and $V_{\hat{y}}$, the quantity $\omega \pm \frac{1}{2} H$ in (3.6) is deformed by purely $(3,0)$ and $(0,3)$ components $\delta \omega \pm \frac{1}{2} \delta H$. Note that $H$ is automatically antisymmetric in all indices. The definition of $\omega_{a b c}$ guarantees that it is antisymmetric in $b$ and $c$; the fermion couplings in (3.6), (3.9) and (3.10) are consistent with this.

We have not performed the analogous computation for the universal hypermultiplet $y_{\phi}$. Instead, will deduce the corresponding auxiliary field from spacetime considerations in Secs. 4 and 5.

\subsection{Target space interpretation}

Following [1]:2], our goal is to interpret the results of the previous subsection in terms of known fluxes and target space structures. We will begin by organizing the results according to the amount of broken worldsheet supersymmetry. At the end of this subsection we will then identify the auxiliary fields in terms of intrinsic torsion classes for string backgrounds with local $S U(3) \times S U(3)$ structure.

The basic results are as follows. We wish to describe independent deformations of the two auxiliary fields $y, \hat{\bar{y}}$. In type IIA compactifications, $y$ and $\hat{\bar{y}}$ can be independently 
tuned, while staying within the class of manifolds with $S U(3)$ structure and $H$-flux of the type $W_{3,4}$ in (2.10) and $H_{3,4}$ in (2.11). This is because the torsion and $H$-flux can be adjusted independently, and the $H$-flux couples with opposite sign to left- and right-moving worldsheet fermions.

However, for type IIB compactifications, we will see that there are a class of auxiliary fields for which the $H$-flux does not contribute; the deformations are entirely geometric. The fields $y, \hat{\bar{y}}$ must correspond to different geometric structures coupling to the left and right movers. Each defines an $S U(3)$ structure. Thus, we expect that the compactifications with $y, \hat{\bar{y}} \neq 0$ are locally manifolds with $S U(3) \times S U(3)$ structure. On the other hand, geometric deformations couple identically to left and right movers, and the only way to enforce the statement that a given $S U(3)$ structure couples chirally is if the local patches of the compactification are glued together with transformations which act chirally. These transformations cannot be diffeomorphisms - they must involve nontrivial reshufflings of string theoretic degrees of freedom, as does T-duality. Therefore, the generic manifold with either or both of $y, \hat{\bar{y}}$ must be a nongeometric compactification along the lines of [26, 27, 28, 30, 58, 69,.

$\mathcal{N}=(2,2)$ supersymmetry

In case of $\mathcal{N}=(2,2)$ supersymmetry, we require that the spin connection and NS-NS three-form, both with lowered vielbein indices, have no $(3,0)$ or $(0,3)$ components. This follows from the fact that the torsion is a derivative of the metric $g$, with $g \sim \partial \bar{\partial} K$ for a real potential $K$, as required by supersymmetry. Therefore, if $\mathcal{N}=(2,2)$ supersymmetry is to be preserved, a deformation of the form (3.9) must be accompanied by a change in the almost complex structure.

If the deformation contains no $H$ flux, the resulting background is a Calabi-Yau background. If the deformation has $H \neq 0$, then there must be two almost complex structures satisfying (2.5). The resulting six-dimensional background will no longer have $S U(3)$ holonomy, but instead an $S U(3) \times S U(3)$ structure. As discussed in $\S 2.3$, the two almost complex structures satisfying (2.5) allow one to construct two conserved $U(1)_{R}$ charges on the worldsheet, which are part of the $\mathcal{N}=(2,2)$ superconformal algebra.15

15 One could also attempt to construct two non-conserved $U(1)_{R}$ currents, $J_{L, \mu \nu} \psi_{R}^{\mu} \psi_{R}^{\nu}$ and $J_{R, \mu \nu} \psi_{L}^{\mu} \psi_{L}^{\nu}$. These can be bosonized and used to construct additional non-conserved spacetime supercharges in an $N=4$ algebra. We expect the corresponding supersymmetries to be broken at the Kaluza-Klein scale. 
When $\mathcal{N}=(2,2)$ supersymmetry is preserved, as long as the $U(1)_{R}$ charge of physical states remains appropriately quantized, we have not broken the spacetime supersymmetry. Given the assumption that violating the R-charge quantization rule of [49,50] corresponds to turning on auxiliary fields in vector multiplets, backgrounds corresponding to $y \neq 0$ and/or $\hat{\bar{y}} \neq 0$ must correspond to backgrounds with reduced worldsheet supersymmetry.

$\mathcal{N}=(2,1)$ supersymmetry

A background for which $y=0$ and $\hat{\bar{y}} \neq 0$ (and the auxiliary fields of the complex structure moduli vanish) will preserve $\mathcal{N}=1$ spacetime supersymmetry. This requires $\mathcal{N}=(2,1)$ supersymmetry on the worldsheet [49,50].

The Kähler deformations and deformations of the auxiliary fields can be classified according to their representations with respect to the $S U(3)$ structure group. The holomorphic and antiholomorphic indices of tensors on the target space transform in the $\mathbf{3}$ and $\overline{\mathbf{3}}$ representations, respectively, of this $S U(3)$. Kähler deformations of the metric $g_{i \bar{j}}$ preserve the complex index structure. The metric $g_{i j}$ itself transforms as a singlet. Following [10], we can use Lefschetz decomposition to parametrize Kähler deformations according to their $S U(3)$ representations:

$$
\delta g_{i \bar{j}}=t_{\mathbf{1}} g_{i \bar{j}}+\left(K_{\mathbf{8}}\right)_{i \bar{\jmath}} \equiv t_{\mathbf{1}} g_{i \bar{j}}+t_{\mathbf{8}}^{a}\left(\omega_{\mathbf{8} a}\right)_{i \bar{\jmath}} .
$$

Here $t_{\mathbf{1}}$ is a rescaling of the overall volume and transforms as an $S U(3)$ singlet. The primitive 2 -form $K_{\mathbf{8}}$ transforms in the $\mathbf{8}$ of $S U(3)$, and the primitive 2-forms $\omega_{\mathbf{8} a}=$ $\left(\omega_{\mathbf{8} a}\right)_{i \bar{\jmath}} d z^{i} \wedge d \bar{z}^{\bar{\jmath}}$ for $a=1, \ldots, h^{1,1}-1$ are a basis of the elements of $H^{(1,1)}(M)$ which each transform in the $\mathbf{8}$ of $S U(3)$.

The quantity $\Omega_{i j k}$ is an $S U(3)$ singlet. Therefore Eqs. (3.9) and (3.10) imply that the auxiliary fields $y, \hat{\bar{y}}$ transform under the same $S U(3)$ representation as the Kähler deformation in the same supermultiplet.

Let us first consider the auxiliary partners $y_{\mathbf{8}}$ of deformations of type $K_{\mathbf{8}}$. Since $H$ is totally antisymmetric, a totally holomorphic deformation lies in a singlet of $S U(3)$. Therefore $y_{8}$ corresponds to a deformation of the spin connection only. It is clear that the deformation (3.9) will break the left-moving $\mathcal{N}=2$ by breaking the $U(1)_{R}$ charge. However, the spin connection couples with the same sign to both the left- and right-moving fermions. In order that the deformation preserve the right-moving $\mathcal{N}=2$, there must be another complex structure $J_{+}=J_{C Y}+\delta J_{+}$under which the deformation (3.9) is no longer $(3,0)$, and which generates a conserved right-moving $U(1)_{R}$ charge $J_{+}=J_{+\mu \nu} \psi_{+}^{\mu} \psi_{+}^{\nu}$. 
At this point we have a puzzle. If the background described by $y_{8} \neq 0$ is a manifold, nothing prevents us from defining a conserved left moving $U(1)_{R}$ current $\tilde{J}_{-}=J_{+\mu \nu} \psi_{-}^{\mu} \psi_{-}^{\nu}$ and restoring $\mathcal{N}=(2,2)$ worldsheet supersymmetry as well as $\mathcal{N}=2$ spacetime supersymmetry. The only possible problem is if the global structure of the compactification is such that $\tilde{J}_{-}$is not well defined. This requires transition functions on the target space of the sigma model which act differently on the left- and right-moving worldsheet fermions. This is only possible in a locally geometric background if the worldsheet fields describing different geometric patches are related by "stringy" transformations rather than just spacetime diffeomorphisms. When the background is locally a torus fibration, T-duality on the fibers is a classic example of such a transformation. We will sketch a more explicit example below.

In summary, for $y_{\mathbf{8}} \neq 0, \hat{\bar{y}}_{\mathbf{8}}=0$ there are either one or two almost complex structures. The original complex structure generates a left-moving $U(1)_{R}$ that is explicitly broken and may or may not be globally defined when coupled to either the left-movers or the right-movers. There must be a deformed complex structure which is globally well-defined when coupled to the right-movers, and is not globally well-defined when coupled to the leftmovers. The result is a background which is locally described as having an $S U(3) \times S U(3)$ structure; globally it is not a manifold, and the left-moving $U(1)_{R}$ is either broken or not globally defined. This is consistent with the observation in [18] that compactifications on manifolds with $S U(3) \times S U(3)$ structure are typically "nongeometric". Note again that our methods do not seem to include the totally nongeometric flux discussed in [19,20. We believe that this is because we are considering a geometric starting point, and studying small deformations. In this sense, backgrounds that are locally nongeometric should correspond to some kind of large deformation; it would be interesting to make this idea more precise.

Next, consider the auxiliary field $y_{1}$ that is the superpartner of the volume deformation. In this case, the corresponding deformation (3.9) of the worldsheet can be made up of both the spin connection $\omega$ and the torsion $H$. As above, this preserves $N=(2,1)$ supersymmetry if an almost complex structure $J_{+}$exists which is covariantly conserved with respect to the torsionful connection $\Gamma_{-}=\Gamma-\frac{1}{2} H$, (where $\Gamma$ is the Levi-Civita connection) and with respect to which the metric is Hermitian [64,60].

If $H \neq 0$, the right-moving current $\tilde{J}_{-}=J_{+\mu \nu} \psi_{-}^{\mu} \psi_{-}^{\nu}$ will not be conserved; this would require that $\left[\left(\partial+\left(\Gamma+\frac{1}{2} H\right)\right) J_{+}\right]_{\mu \nu \lambda}=0$, which is incompatible with the left-moving current being conserved. In this case it is possible that the background is globally a 
manifold with $S U(3) \times S U(3)$ structure, without nongeometric features. Alternatively, $\tilde{J}_{-}$ may not be globally well-defined, and we have the same situation as $\mathbf{y}_{\mathbf{8}} \neq 0$ discussed above.

$\mathcal{N}=(1,1)$ supersymmetry

Worldsheet supersymmetry by itself imposes no serious constraints in this case. The deformations can lie in the subset of deformations with $S U(3)$ structure, as long as the complex structure is not conserved with either connection $\Gamma_{ \pm}$. To be able to tune $y, \hat{\bar{y}}$ independently, we must be in the class of compactifications that have a local $S U(3) \times S U(3)$ structure; for $y_{\mathbf{8}}, \hat{\bar{y}}_{\mathbf{8}}$ to be independently tuneable, the backgrounds must presumably be globally nongeometric.

\section{Auxiliary fields and torsion classes}

We have shown that general values of $y, \hat{\bar{y}}$ correspond to compactifications with local $S U(3) \times S U(3)$ structure. The coupling of the left- and right-moving fermions will be described by the almost complex structures $J_{ \pm}$defining the two $S U(3)$ structures independently. Using Eqs. (2.15), (3.9), and (3.10), we find that

$$
\begin{aligned}
& \bar{W}_{1}^{1}+3 i \bar{H}_{1}^{1}=y_{\mathbf{1}} \\
& \bar{W}_{2, a \bar{b}}^{1}=-8 i \sum_{I} y_{\mathbf{8}}^{I} \omega_{a \bar{b}}^{I}
\end{aligned}
$$

and

$$
\begin{aligned}
& W_{1}^{2}+3 i H_{1}^{2}=\hat{\bar{y}}_{\mathbf{1}} \\
& W_{2, a \bar{b}}^{2}=-8 i \sum_{I} \hat{\bar{y}}_{\mathbf{8}}^{I} \omega_{a \bar{b}}^{I}
\end{aligned}
$$

Note that since we are taking our auxiliary fields to be constant vevs, these equations imply that $W_{1}$ and $H_{1}$ are constant. This seems to imply, by (2.10), that $J \wedge J$ is exact (when all the other torsion classes are turned off), which is not true for compact CalabiYau backgrounds. In a noncompact model, $J \wedge J$ can be exact (for example, in flat space). At any rate we only expect to be allowed to turn on a small amount of torsion and flux in noncompact models. 


\subsection{SU(3) structure compactifications}

String compactifications on manifolds with $S U(3)$ structure have been intensively studied in the last several years. Here we see how these fit into our framework. In these cases, $W_{k}^{1}=W_{k}^{2}=W_{k}$, and $H_{k}^{1}=H_{k}^{2}=H_{k}$. We can see instantly from Eqs. (3.13) and (3.12) that this means that $y_{\mathbf{8}}, \hat{\bar{y}}_{\mathbf{8}}$ are complex conjugates.

Because one can deform the metric and the $H$-flux independently, we may still sepa-

rately dial $y_{1}, \hat{\bar{y}}_{1}$. One might be tempted to conclude that since we can set $y_{1} \neq 0, \hat{\bar{y}}_{1} \neq 0$, there is a class of $S U(3)$ structure compactifications with $W_{1} \neq 0$ which is compatible with spacetime supersymmetry. However, we will see in $\S 4$ that the auxiliary field for the universal (dilaton) hypermultiplet is a different linear combination of $W_{1}$ and $H_{1}$. Therefore, if $y_{1} \neq 0$ and $\hat{\bar{y}}_{1}=0$, the auxiliary field $\hat{\bar{y}}_{\phi}$ in the universal hypermultiplet will still be nonzero. This matches the known fact that half-flat manifolds in type IIB are incompatible with spacetime supersymmetry (see [70] and references therein).

\subsection{Nongeometric compactifications}

We have shown that typical compactifications with $y_{\mathbf{8}} \neq 0$ should have "nongeometric" features, in which the transition functions on different coordinate patches act chirally on the fermions. Our argument was based on the requirement that despite the existence of a well-defined left-moving $U(1)_{R}$ current constructed from an almost complex structure, there should be no well-defined right-moving $U(1)_{R}$ current constructed from the same almost complex structure. We will sketch a scenario in which this can occur, to make our reasoning clearer.

Consider a compactification that is locally a complex manifold with a Lagrangian $T^{3}$ fibration. This fibration will have monodromies as one encircles singular loci of the fibration on the base $B$ [16,71, 72]. For a purely geometric fibration, such as a Calabi-Yau [71] or a manifold with "geometric flux" [16, 20,73, ,74, the monodromy will lie in the group $G L(3, \mathbb{Z})$ of discrete diffeomorphisms.

More generally, this monodromy can lie in the full duality group of $T^{3}$. We will restrict ourselves (arbitrarily) to the perturbative duality group $O(3,3 ; \mathbb{Z})$. Such backgrounds are often known as "T-folds"; various examples have been discussed in [26-31]

Imagine that the monodromy about some loop is a non-trivial T-duality acting on two 1-cycles of the torus, much as in the example discussed in [73]. Let the coordinates $y^{i}$ be the coordinates on the $T^{3}$ and $x^{i}$ be coordinates on the base $\mathcal{B}_{3}$, such that $z^{i}=x^{i}+i y^{i}$, 
for $i=1,2,3$. If the T-duality in the monodromy discussed above acts on $y^{2,3}$, then it will act on the fermions as

$$
\psi_{ \pm}^{y^{2,3}} \rightarrow \pm \psi_{ \pm}^{y^{2,3}}
$$

and all other worldsheet fermions lying along the compactification directions will not transform. If we write the fermions in complex coordinates, then the monodromy will act as:

$$
\begin{gathered}
\psi_{+}^{z^{i}} \rightarrow \psi_{+}^{z^{i}} \\
\psi_{-}^{z^{1}} \rightarrow \psi_{-}^{z^{1}} \\
\psi_{-}^{z^{2,3}} \rightarrow \psi_{-}^{\bar{z}^{2,3}}
\end{gathered}
$$

Now, suppose that

$$
J_{+}=\sum_{i=1}^{3} \psi_{+}^{z^{i}} \psi_{+}^{\bar{z}^{i}}
$$

is a globally well defined operator - in other words, it will be preserved under monodromy transformations arising from loops in $\mathcal{B}_{3}$ about the singular loci of the fibration. (The operator is certainly invariant under the monodromy action above). It may seem natural to define a left-moving $U(1)_{R}$ current

$$
J_{-}=\sum_{i=1}^{3} \psi_{-}^{z^{i}} \psi_{-}^{\bar{z}^{i}}
$$

but this is not globally well-defined, as the monodromy action on $J_{-}$is

$$
J_{-}=\psi_{-}^{z^{1}} \psi_{-}^{\bar{z}^{1}}+\psi_{-}^{z^{2}} \psi_{-}^{\bar{z}^{2}}+\psi_{-}^{z^{3}} \psi_{-}^{\bar{z}^{3}} \rightarrow \psi_{-}^{z^{1}} \psi_{-}^{\bar{z}^{1}}-\psi_{-}^{z^{2}} \psi_{-}^{\bar{z}^{2}}-\psi_{-}^{z^{3}} \psi_{-}^{\bar{z}^{3}}
$$

In general, only a monodromy action which includes a T-duality transformation will act chirally on the fermions in this way. A more global analysis is required to see if a different left-moving $U(1)_{R}$ charge is globally defined, and whether it is conserved; if no such conserved current exists, then one can have an $\mathcal{N}=(1,2)$ compactification without $H$-flux, as seems to appear when only the $y_{\mathbf{8}}$ auxiliary fields are turned on.

\subsection{Mirror symmetry}

One goal of this paper is to understand mirror symmetry for compactifications with H-flux. Here we argue that our results confirm previous statements [11, 10, 19, 20] that the mirrors of such compactifications are compactifications with local $S U(3) \times S U(3)$ structure, which are often T-folds. 
The first argument arises from the four-dimensional effective action. Consider a type IIA compactification with nonvanishing auxiliary fields for the hypermultiplets, which are complex structure deformations. In [1,2], these auxiliary fields were shown to be combinations of $H$-flux of type $H_{3,4}$ and torsion of type $W_{3,4}$, according to the definitions (2.10) and (2.11). Mirror symmetry should leave the variables of the four-dimensional effective action invariant. Thus, we expect that the mirror of such combinations of flux and torsion to be auxiliary fields of type $y_{1}, y_{\overline{8}}$, which are related to the Kähler moduli.

The essence of this argument is that if mirror symmetry holds for Calabi-Yau backgrounds, it holds for deformations of Calabi-Yau backgrounds, as long as one understands the mirror map acting on deformations of the theory. Of course this is dangerous; we are assuming that compactifications of the type we care about can be considered as connected in field space to an $\mathcal{N}=2$ compactification, and that mirror symmetry remains valid for off-shell deformations. Since we expect mirror symmetry to hold for all correlation functions (which can be used to define an effective potential, at least in a power series about a given point in field space) the assumption is not obviously wrong.

The second argument arises from the worldsheet point of view. From this standpoint the reader might sensibly object that the cases we have in mind have at most $\mathcal{N}=1$ spacetime supersymmetry and therefore at most $\mathcal{N}=(2,1)$ worldsheet supersymmetry. The conformal field theory explanation of mirror symmetry uses the structure of the $\mathcal{N}=$ $(2,2)$ superconformal algebra: namely, one simply reverses the sign of the right-moving $U(1)_{R}$ current relative to the left moving $U(1)_{R}$ current.

However, we do not believe that this poses an obstacle to defining mirror symmetry from the worldsheet point of view, at least if the IIA compactification is a manifold with $H$ flux. Such type IIA compactifications with $y \neq 0$ still have a right-moving $U(1)_{R}$ current $\tilde{J}_{+}$which exists but is not conserved: take the almost complex structure $J_{-\mu \nu}$ used to define the left-moving $U(1)_{R}$ current, and write $\tilde{J}_{+}=J_{-\mu \nu} \psi_{+}^{\mu} \psi_{+}^{\nu}$. It still makes sense to reverse the sign of this non-conserved $U(1)_{R}$. In the IIB mirror, while the local almost complex structure defining the left moving $U(1)_{R}$ cannot be used to construct a globally well defined right-moving $U(1)_{R}$ current, there would be a different local almost complex structure which leads to a globally defined but nonconserved right-moving $U(1)_{R}$ current. 


\section{Ten-dimensional supergravity calculation of auxiliary fields}

In this section, we will calculate the auxiliary fields using ten dimensional supergravity, by studying the supersymmetry variations of the fermions in the hypermultiplets directly. The four-dimensional supersymmetry transformations are (2.3), with $\eta^{N}$ the nowherevanishing spinors defining the $S U(3) \times S U(3)$ structure, and $\eta_{1}=\eta_{2}$ for Calabi-Yau backgrounds. For the Kähler moduli, this calculation should be essentially identical to the calculations in Sec. 3 -after all, the formula (3.4) essentially implements two spacetime supersymmetry transformation on the worldsheet vertex operator for the scalar in the multiplet [65, 66]. One difference will be that in this section we also consider auxiliary fields for the universal hypermultiplet, with a superfield expansion as given in (2.1). It would be worthwhile to do the same calculation for the auxiliary fields that arise in the presentation of [42], or for other off-shell hypermultiplets. Additionally, now that we are no longer confined to a worldsheet description, one could include RR fluxes (as in [10]). We expect these fields will show up as additional contributions to the auxiliary fields; an argument that the RR 0-form appears in such a manner for vector multiplets was given in [1,2]. They will also appear in other off-shell representations of the hypermultiplets. We leave this exercise for future work.

We now proceed to calculate $y_{\mathbf{1}}, y_{\mathbf{8}}$, and the auxiliary field $y_{\phi}$ for the universal hypermultiplet, in turn.

\subsection{Auxiliary field for the volume deformation $y_{1}$}

For a general Kähler deformation (3.11) of the Calabi-Yau metric away from a fixed metric $g_{i \bar{j}}$, the rescaling $t_{\mathbf{1}}$ of the volume can be picked out by contracting $\delta g$ with $g$,

$$
t_{\mathbf{1}}=\frac{1}{3} g^{i \bar{j}} \delta g_{i \bar{j}} .
$$

Since the trace will select out a variation that is proportional to $\delta g$, it will be independent of the internal coordinates.

We will work in ten-dimensional string frame, to more easily match the worldsheet calculation of the previous section. The supersymmetry variation of $\delta g$ implies that the fermionic superpartner is:

$$
\chi_{\mathbf{1} \pm}^{N}=\frac{i}{3}\left(\eta_{ \pm}^{N}\right)^{\dagger} \gamma^{\bar{i}} \Psi_{\bar{i}}^{N}
$$


where the \pm subscripts for $\chi$ denote four-dimensional chirality, $\Psi_{m}^{N}$ are the two tendimensional gravitinos polarized along $M$, and we have used $\delta_{\epsilon^{N}}\left(\delta g_{m n}\right)=i \bar{\epsilon}^{N}\left(\Gamma_{m} \Psi_{n}^{N}+\right.$ $\left.\Gamma_{n} \Psi_{m}^{N}\right)$. The bosonic part of the supersymmetry variation of $\chi_{\mathbf{1}}$ will come entirely from the supersymmetry variation of $\Psi_{m}$ in $(4.2)$. These are given in [75, ,76]. 16 . Let $y_{1}^{N=1}=y_{1}$ and $y_{\mathbf{1}}^{N=2}=\hat{\bar{y}}_{\mathbf{1}}$. The supersymmetry variation of $\psi_{\mathbf{1},+}$ with respect to an infinitesimal parameter $\zeta$ is:

$$
\begin{aligned}
\delta \chi_{\mathbf{1}+}^{N} & =\zeta_{N+} \otimes y_{\mathbf{1}}^{N}+\ldots \\
& =\frac{i}{3} \zeta_{N+} \otimes\left(\eta_{+}^{N}\right)^{\dagger} \gamma^{m}\left(D_{m}+(-1)^{N} \frac{1}{8} H_{m B C} \gamma^{B C}\right) \eta_{-}^{N}+\cdots
\end{aligned}
$$

where $D_{m} \eta=\partial_{m} \eta+\frac{1}{4} \omega_{m A B} \gamma^{A B} \eta$ and capital letters $A, B, \ldots$ run over all six internal indices.

Now, using (2.7), we find that

$$
y_{\mathbf{1}}=\frac{i}{3}\left[i q_{m n}\left(\eta_{+}^{\dagger} \gamma^{m} \gamma^{n} \eta_{+}\right)-\frac{1}{8} \eta_{+}^{\dagger} \gamma^{m} \gamma^{B C} H_{m B C} \eta_{-}\right]
$$

The specific expressions for $q$ are given in [13, 15]. $\left(q_{m}, \tilde{q}_{m}\right.$ do not contribute because they multiply the wrong chirality). Using (2.8), the first term on the left hand side of (4.4) will project on the $S U(3)$ singlet part of $q_{m n}$. The result, combined with (2.9), confirms (3.12), (3.13) precisely for $y_{1}, \hat{\bar{y}}_{1}$, up to an overall prefactor.

\subsection{Auxiliary fields y8 for remaining Kähler moduli}

For a general deformation of the metric $\Delta g_{m n}$ polarized along the internal directions, the fermion partner under the supersymmetry deformation can be computed using the ten-dimensional supersymmetry algebra:

$$
\chi_{(m n), \pm}^{N}=\left(\eta_{ \pm}^{N}\right)^{\dagger}\left(\gamma_{m} \Psi_{n}^{N}+\gamma_{n} \Psi_{m}^{N}\right)
$$

Note that these are not yet four-dimensional fields in the usual sense; $\Delta g$ and $\psi$ depend on the coordinates of $M$. For a particular four-dimensional scalar arising from reducing the metric on a particular internal wavefunction, we would reduce the fermion as well.

To find the auxiliary field $y$ for such a deformation, we compute $\delta \chi_{+}^{1}$ and find that:

$$
y_{(m n)}^{1}=\left(i \delta_{(m}^{p}-J_{(m}^{p}\right) q_{n) p}-i \Omega_{(m}^{p q} H_{n) p q}
$$

16 Note that the subscripts \pm in Appendix B of Ref. [76 label the two supersymmetries of type IIB supergravity. 
Again, $y_{(m n)}^{1}$ as defined is not yet a four-dimensional field in the usual sense.

Now, if we are interested in the auxiliary fields for $\Delta g_{i \bar{j}}=t_{\mathbf{8}}^{I} \omega_{i \bar{j}}^{I}$, with $\omega^{I}$ a basis of primitive harmonic $(1,1)$ forms, we should expand $y^{1}=y$ in the same basis. Following [13,15], $H$ has terms transforming in the $1, \underline{3}, \mathbf{6}$, and conjugates, and will not contribute. The only term in $q_{m n}$ transforming in the $\mathbf{8}$ is proportional to $W_{2}$, and the result confirms (3.12), (3.13) for $y_{8}$.

\subsection{Auxiliary field $y_{\phi}$ for the universal hypermultiplet}

The real part of the scalar field in the universal hypermultiplet is the four-dimensional

dilaton $\phi_{4}=\phi_{10}-\frac{1}{2} \ln \mathcal{V}$. Since $\Delta \mathcal{V}=3 \Delta t_{1} \mathcal{V}$, we find that the four-dimensional dilatino is, in string frame

$$
\lambda_{ \pm}^{\phi_{4}, N}=\left(\eta_{ \pm}^{N}\right)^{\dagger} \lambda^{\phi_{10}, N}-\frac{3}{2} \chi_{\mathbf{1}, \pm}^{N}
$$

Using the variations given in [75, 76], we find that

$$
\begin{array}{r}
y_{\phi_{4}}=-\frac{3 i}{4}\left(\bar{W}_{1}^{1}+i \bar{H}_{1}^{1}\right) \\
\hat{\bar{y}}_{\phi_{4}}=-\frac{3 i}{4}\left(W_{1}^{2}-i H_{1}^{2}\right)
\end{array}
$$

As an application of this result, recall that in $\S 3.4$, we commented that even though one can set $y_{1}=0$ in the set of compactifications with $S U(3)$ structure (for which $H_{1}^{1}=$ $\left.H_{1}^{2}=H_{1}, W_{1}^{1}=W_{1}^{2}=W_{1}\right)$, we expect that supersymmetry is broken if $W_{1}, H_{1} \neq 0$. This is because $y_{1}$ and $y_{\phi_{4}}$ are independent linear combinations of $\bar{W}_{1}, \bar{H}_{1}$, and can both vanish only if $W_{1}, H_{1}=0$.

This is very close to the results in [52,53, which find that the F-term for the dilaton is a combination of the $(3,0)$ components of the NS-NS and R-R 3-form field strengths. We find a purely NS-NS deformation because we are studying auxiliary fields which break a different $\mathcal{N}=1$ subgroup of the $\mathcal{N}=2$ supersymmetry.

\section{Four-dimensional Supergravity calculation of auxiliary fields}

In this section we check our results in $§ 3.4$ against the superpotential for $S U(3)$ structure compactifications proposed by [10]. Closely related and complementary results were obtained in [63]. 


\subsection{Superpotential for $S U(3)$ structure compactifications}

Graña et. al. [10] computed the $\mathcal{N}=2$ Killing prepotential and the superpotential for an arbitrary $\mathcal{N}=1$ subalgebra of the $\mathcal{N}=2$ symmetry of the effective action, for compactifications with $S U(3) \times S U(3)$ structure. We will specialize to the case of $S U(3)$ structure with one or more of $\mathcal{W}_{1,2}$ and $H_{1} \neq 0$, and focus on the $\mathcal{N}=1$ subalgebras generated by the left- and right-movers on the worldsheet.

As we have pointed out in the previous two sections, $S U(3)$ structure compactifications of this type break supersymmetry completely. In order to check our previous formulae, we will choose one of the $\mathcal{N}=1$ subalgebras and use the results of [10], who extract the corresponding superpotential from the prepotentials. For example, we can choose a superpotential $W$ for the $\mathcal{N}=1$ supersymmetry corresponding to the superspace directions $\theta$; this implies a nonvanishing expectation value of $\hat{\bar{y}}$. The auxiliary field $y$ of the $\mathcal{N}=2$ hypermultiplet becomes the auxiliary field for the $\mathcal{N}=1$ chiral multiplet

$$
w^{a}+\theta \chi^{a}+\theta^{2} y^{a}
$$

We can determine $y$ by computing the Kähler covariant derivative of $\bar{W}$ :

$$
F^{A}=-e^{K / 2 m_{p, 4}^{2}} K^{a \bar{b}} D_{\bar{b}} \bar{W},
$$

where $K$ is the Kähler potential, and $K^{a \bar{b}}$ is the inverse of $K_{a \bar{b}}=\partial_{a} \partial_{\bar{b}} K$, and $m_{p, 4}$ is the four-dimensional Planck mass. The Kähler covariant derivative is:

$$
D_{a} W=\partial_{a} W+\frac{1}{m_{p, 4}^{2}} W \partial_{a} K .
$$

The results of [10] can be summarized as follows. Let us denote Kähler and complex structure moduli by $t$ and $u$, respectively. Define

$$
W(t, u)=i m_{p, 4}^{3} \int(B+i J) \wedge d \Omega \quad \text { and } \quad \tilde{W}(\bar{t}, u)=-i m_{p, 4}^{3} \int(B-i J) \wedge d \Omega .
$$

where we assume that all geometric quantities in the integral are given in string units, and the dimensionful factor in front is the correct one for the four-dimensioinal effective action (cf. the appendix of [77]). 1 . Specializing to the case of $S U(3)$ structure, the results

17 In our noncompact case, one might worry about the convergence of (5.4). To avoid such problems, we should think cutting off the large-volume part of our Calabi-Yau and gluing it into a compact space, taking appropriate care with boundary conditions. We leave such questions to future work. 
of [10] (cf. Eqs. (2.148) and (2.149)) imply that the $\mathcal{N}=1$ superpotentials for the $\mathcal{N}=1$ subalgebras generated by left- and right-moving supersymmetries, respectively, are:

$$
W_{\mathrm{IIB}}(t, u)=W(t, u), \quad \hat{W}_{\mathrm{IIB}}(\bar{t}, u)=\tilde{W}(\bar{t}, u) .
$$

These can be checked by matching the coefficients of the H-flux in the gravitino and dilatino variations, between variations of the string frame fields in [75,76] on the one hand and the variations of the Einstein frame fields in [10].

For pertubations away from a Calabi-Yau geometry, these formulae deserve a word of interpretation. We wish to split variations of $B, J$ into:

$$
\begin{gathered}
B=B_{C Y}+B_{t} \\
J=J_{C Y}+J_{t}
\end{gathered}
$$

Here $J_{C Y}, B_{C Y}$ correspond to the Kähler form and NS-NS 2-form for the underlying CalabiYau compactification - in particular we take them to carry all of the dependence on the Kähler moduli, and $d B_{C Y}=d J_{C Y}=0 . B_{t}, J_{t}$ are not closed $-d B_{t}=H$, and $d J_{t}$ is a proportional to the intrinsic torsion. Similarly, we will write $\Omega=\Omega_{C Y}+\Omega_{t}$, where $\Omega_{C Y}$ is closed and $d \Omega_{t}$ is proportional to various intrinsic torsion classes. In particular, if we assume the intrinsic torsion and $H$ are small, then we can write

$$
\begin{aligned}
& d J_{t}=\frac{3 i}{4}\left(W_{1} \bar{\Omega}_{C Y}-\bar{W}_{1} \Omega_{C Y}\right)+W_{4} \wedge J_{C Y}+W_{3} \\
& d B_{t}=H_{t}=\frac{3 i}{4}\left(H_{1} \bar{\Omega}_{C Y}-\bar{H}_{1} \Omega_{C Y}\right)+H_{4} \wedge J_{C Y}+H_{3} \\
& d \Omega_{t}=W_{1} J_{C Y}^{2}+W_{2} \wedge J_{C Y}+\bar{W}_{5} \wedge \Omega_{C Y} .
\end{aligned}
$$

Note that this decomposition into a background and deformation is only valid for a noncompact Calabi-Yau, where we are allowed to locally turn on small amounts of torsion. In general, such a decomposition would not be sensible.

Let us consider the $\mathcal{N}=1$ algebra generated by the left-movers, with superpotential $W$. The $\mathcal{N}=1$ Kähler potential is the sum of terms $K_{1}, K_{2}$ and $K_{3}$ for the Kähler moduli, complex structure moduli and dilaton, respectively. The Kähler potential for Kähler moduli is:

$$
e^{-K_{1} / m_{p, 4}^{2}}=\frac{4}{3} \int J \wedge J \wedge J=8 V,
$$

where $V$ is the volume of $M$ in string units. The Kähler potential for complex structure moduli is

$$
e^{-K_{2} / m_{p, 4}^{2}}=i \int \Omega \wedge \bar{\Omega}=8 V .
$$


where $\Omega$ is the canonically normalized (3,0)-form $\left(\Omega_{\eta}\right.$ in the notation of Ref. [10] 10 Finally, the Kähler potential for the four-dimensional dilaton is:

$$
e^{-K_{3} / m_{p, 4}^{2}}=e^{-2 \phi_{4}}=-\frac{i}{2}(\tau-\bar{\tau}),
$$

where $\tau=a+i e^{-2 \phi_{4}}$ '; $a$ is the model-independent NS-NS axion dual to the NS-NS 2-form in four dimensions; and $\phi_{4}$ is the four-dimensional dilaton.

\subsection{Overall volume modulus: 1 of $S U(3)$}

We consider the metric deformation $t_{\mathbf{1}}$ in the $\mathbf{1}$ of $S U(3)$ : that is, $\delta J=t_{\mathbf{1}} J$. The complex scalar in the chiral multiplet can be written as $w=b+i t_{\mathbf{1}}$, where $B=b J$. If we write $V=V(w-\bar{w})$, then

$$
\left.\partial_{w} V\right|_{w=0}=-\left.\partial_{\bar{w}} V\right|_{w=0}=-\frac{3 i}{2} V .
$$

The relevant derivatives of the Kähler potential are:

$$
\begin{gathered}
K_{1, \bar{w}}=-m_{p, 4}^{2} \partial_{\bar{w}} \ln V=-\frac{3 i}{2} m_{p, 4}^{2} \\
K_{1, w \bar{w}}=-m_{p, 4}^{2} \partial_{w} \partial_{\bar{w}} \ln V=\frac{9}{4} m_{p, 4}^{2}
\end{gathered}
$$

We wish to calculate $y_{1}$, using (5.2) with $W=W_{I I B}(t, u)$. The Kähler covariant derivative of $\bar{W}$ is:

$$
\begin{aligned}
D_{\bar{w}} \bar{W} & =-i m_{p, 4}^{3} \partial_{\bar{w}} \int\left(B_{C Y}-i J_{C Y}\right) \wedge d \bar{\Omega}_{t}+\frac{3}{2} m_{p, 4}^{3} \int\left(H_{t}-i d J_{t}\right) \wedge \bar{\Omega}_{C Y} \\
& =-i m_{p, 4}^{3} \int J_{C Y} \wedge d \bar{\Omega}_{t}+\frac{3}{2} m_{p, 4}^{3} \int\left(H_{t}-i d J_{t}\right) \wedge \bar{\Omega}_{C Y} \\
& =-i \bar{W}_{1} \int J^{3}+\frac{3}{2}\left(\frac{-3 i}{4}\right)\left(\bar{H}_{1}-\bar{W}_{1}\right) \int \Omega_{C Y} \wedge \bar{\Omega}_{C Y} \\
& =a V\left(\bar{W}_{1}+3 i \bar{H}_{1}\right)
\end{aligned}
$$

where we have used $J_{C Y}^{3}=\frac{3 i}{4} \Omega_{C Y} \wedge \bar{\Omega}_{C Y}$ (cf. [10]), and where $a$ is a numerical constant. The resulting auxiliary field is:

$$
y^{w} \equiv F^{w} \propto e^{\phi_{4}} m_{p, 4}\left(\bar{W}_{1}+3 i \bar{H}_{1}\right)=m_{s}\left(\bar{W}_{1}+3 i \bar{H}_{1}\right) .
$$

This matches (3.12). Note the explicit factor of the string mass $m_{s}$. The auxiliary partner $F$ of a dimensionless scalar will have mass dimension 1 . The discussion of $\S 3, \S 4$ was entirely in string frame; all of the lengths were measured in string frame. The dimensions of superpartners arise from explicit powers of $m_{s}$ that appear in the spacetime superalgebra in string frame.

18 Canonically, $\frac{1}{6} J^{3}=\frac{i}{8} \Omega \wedge \bar{\Omega}=\mathrm{Vol}_{6}$, where $\mathrm{Vol}_{6}$ is the volume form. In the framework of Ref. [10], the Kähler and complex structure pure spinors are $\Omega_{+}=c e^{-B-i J}$ and $\Omega_{-}=n \Omega$. 


\subsection{Primitive Kähler moduli: 8 of $S U(3)$}

We now consider arbitrary Kähler moduli $w^{a}$ in the 8 of $S U(3)$, defined by $B+i J=$ $\sum_{a} w^{a} \omega^{a}$, where $\omega^{a}$ is a basis of $H^{1,1}$. The derivatives of the Kähler potential are:

$K_{a}=-\frac{i m_{p, 4}^{2}}{4 V} \int \omega_{a} \wedge J^{2}, \quad K_{a \bar{b}}=-\frac{m_{p, 4}^{2}}{4 V} \int \omega_{a} \wedge \omega_{b} \wedge J+\frac{m_{p, 4}^{2}}{16 V^{2}}\left(\int \omega_{a} \wedge J^{2}\right)\left(\int \omega_{b} \wedge J^{2}\right)$,

and the derivative of the superpotential is:

$$
\partial_{a} W=i m_{p, 4}^{3} \int \omega_{a} \wedge d \Omega=i m_{p, 4}^{3} \int \omega_{a} \wedge\left(W_{2} \wedge J+W_{1} J^{2}\right) .
$$

For Kähler moduli $w^{a}$ in the $\mathbf{8}$ of $S U(3)$, the corresponding $(1,1)$ forms $\omega_{a}$ are primitive. so that $\omega_{a} \wedge J^{2}=0$. In this case,

$$
K_{a}=0, \quad K_{a \bar{b}}=-\frac{m_{p, 4}^{2}}{4 V} \int \omega_{a} \wedge \omega_{b} \wedge J \quad \text { and } \quad \partial_{a} W=i m_{p, 4}^{3} \int \omega_{a} \wedge W_{2} \wedge J .
$$

Writing $\bar{W}_{2}=\bar{W}_{2}^{b} \omega_{b}$,

$$
D_{\bar{a}} \bar{W}=4 i V K_{\bar{a} b} m_{p, 4} \bar{W}_{2}^{b} .
$$

so that

$$
y^{a}=c_{\mathbf{8}} e^{\phi_{4}} m_{p, 4} \bar{W}_{2}^{a}=c_{8} m_{s} \bar{W}_{2}^{a} .
$$

where $c_{8}$ is some complex numerical coefficient. Similarly,

$$
\hat{\bar{y}}^{a}=c_{8}^{*} m_{s} W_{2}^{a} .
$$

This confirms the results given in $\S 3$ and $\S 4$.

\subsection{Universal hypermultiplet}

Next, let us describe the auxiliary fields of the universal hypermultiplet. Since Eq. (5.4) is independent of the dilaton, we have $D_{\tau} W=K_{\tau} W$ proportional to the superpotential. In type IIB, we find that

$$
\begin{aligned}
& y^{\tau}=c_{\phi, 1} e^{-\phi_{4}} m_{p, 4}\left(\bar{H}_{1}-i \bar{W}_{1}\right), \\
& \hat{\bar{y}}^{\tau}=c_{\phi}^{*} e^{-\phi_{4}} m_{p, 4}\left(H_{1}-i W_{1}\right) .
\end{aligned}
$$

where $c_{\phi}$ is a complex numerical constant. Now to compare this to previous sections, we need to transform $y^{\tau}$ to $y^{\phi}$, using $\phi=-\frac{1}{2} \ln [(\tau-\bar{\tau}) / 2 i]$. We find that

$$
\begin{aligned}
& y^{\tau}=\tilde{c}_{\phi} m_{s}\left(\bar{H}_{1}-i \bar{W}_{1}\right), \\
& \hat{\bar{y}}^{\tau}=\tilde{c}_{\phi}^{*} m_{s}\left(H_{1}-i W_{1}\right) .
\end{aligned}
$$

where $\tilde{c}_{\phi}$ is some complex numerical coefficient. This matches the result in $\S 4.3$. 


\subsection{Complex structure moduli: 6 of $S U(3)$}

Finally, in order to tie the language of $S U(3)$ structures to previous work [1,2], we will also compute the NS-NS auxiliary fields for the type IIB complex structure moduli.

Refs. [1,2] showed that the auxiliary fields $D_{++}$corresponded to $H$ and $d J$ lying in $H^{(2,1)}$. From the complex structure moduli $u^{A}$ in the $\mathbf{6}$ of $S U(3)$, we define a basis of primitive $(2,1)$ forms

$$
\chi_{A}=D_{A} \Omega=\left(\partial_{A}+K_{A}\right) \Omega .
$$

$H$ and $d J$ can be expanded in this basis, as we will do.

In terms of the $\chi_{A}$ and their complex conjugates, the metric $K_{\bar{A} B}$ in the complex structure moduli space is

$$
K_{\bar{A} B}=\bar{\partial}_{\bar{A}} \partial_{B} K=-\frac{\int \chi_{B} \wedge \bar{\chi}_{\bar{A}}}{\int \Omega \wedge \bar{\Omega}}=-\frac{i}{8 V} \int \chi_{B} \wedge \bar{\chi}_{\bar{A}} .
$$

Writing the real 3-forms $H_{3}$ and $W_{3}$ as

$$
H_{3}=H_{3}^{A} \chi_{A}+\bar{H}_{3}^{\bar{A}} \bar{\chi}_{\bar{A}} \quad \text { and } \quad W_{3}=W_{3}^{A} \chi_{A}+\bar{W}_{3}^{\bar{A}} \bar{\chi}_{\bar{A}}
$$

we can use (5.23), (5.2), and (5.4), to show that:

$$
D_{++}^{A}=c_{6} m_{s}\left(\bar{H}_{3}^{A}-i \bar{W}_{3}^{A}\right) \quad \text { and } \quad \hat{D}_{--}^{A}=c_{6} m_{s}\left(\bar{H}_{3}^{A}+i \bar{W}_{3}^{A}\right)
$$

for the NS-NS auxiliary fields in the vector multiplets, where $c_{6}$ is a complex numerical constant. This is in precise agreement with the results in [1].2].

\section{Worldsheet instanton corrections}

\subsection{General remarks}

Supergravity arguments have indicated that the mirror of compactifications with $W_{3}, H_{3} \neq 0$ involves intrinsic torsion classes in a locally $S U(3) \times S U(3)$ structure background [10, 11, 18]. While this identification is surely correct, we expect that supergravity will be a poor approximation for such compactifications.19

19 The astute reader will sensibly complain that this has not stopped us from doing supergravity calculations either. Again, for local models we have some hope of being on good footing; beyond that, we should start from the worldsheet discussion in $\S 3$. 
One reason is that, as we have argued in $\S 3$, nongeometric fluxes are generic features of the compactification. The second reason arises from contemplating mirror symmetry in its traditional setting, type II compactifications on Calabi-Yau backgrounds. For compact models mirror symmetry only makes sense when worldsheet instantons are includedindeed, the ability to compute instanton effects is to a large extent what made mirror symmetry so exciting in the first place.

More precisely, let us consider type IIA string theory with expectation values for auxiliary fields in the hypermultiplets. These will be described by NS-NS flux and torsion of type $H_{3}$ and $W_{3}$ which, as mentioned in $\S 5.5$, leads to a superpotential for complex structure moduli. For a compact model the minimum of the corresponding potential is generically deep in the interior of complex structure moduli space. If mirror symmetry is at all valid, the mirror should have volumes of order the string scale, for which nonperturbative worldsheet physics should become important.

Furthermore, recall that in local (noncompact) models, the superpotential for complex structure moduli arises as a term breaking $\mathcal{N}=2$ supersymmetry to $\mathcal{N}=1$ supersymmetry. For vector multiplet moduli, one expands the prepotential to first order in the nonvanishing auxiliary field, and integrates out the superspace directions for the broken supersymmetry, to obtain the superpotential [1,2, [4,6]. A similar calculation should hold for the hypermultiplet moduli. While we leave this project for future work, we note that the $\mathcal{N}=2$ action for the Kähler moduli will receive worldsheet instanton corrections, and so we expect the superpotential to receive such corrections as well. Indeed, in Ref. [14], Gurrieri and Micu attempted to match the bosonic four-dimensional effective action for type IIB string theory on a half-flat manifold to a type IIA compactification with NS-NS 3 -form flux. Close inspection of this paper reveals that in order for the actions match in detail, coefficients of various terms in the type IIB effective action must include terms from worldsheet instanton corrections.

\subsection{One-instanton contribution to the superpotential}

We wish to show that when one can perturb a Calabi-Yau background to an $S U(3)$ structure background with intrinsic torsion of type $W_{1,2}$, worldsheet instantons contribute to the superpotential (for the $\mathcal{N}=1$ spacetime supersymmetry generated by either the leftor right-moving worldsheet sector), with the one-instanton contribution entering precisely at first order in $W_{1,2}$. 
We begin by reviewing the argument in [78,79], that for string backgrounds with $\mathcal{N}=(2,2)$ worldsheet supersymmetry, the Kähler moduli do not get instanton-generated superpotentials. We will adopt the specific arguments in 80 to our present purposes.

Assume that at least $\mathcal{N}=1$ spacetime supersymmetry is preserved, and arises from the right-moving $\mathcal{N}=2$ worldsheet algebra (this could be secretly $\mathcal{N}=2$ supersymmetric, or the $U(1)_{R}$ charges of the right-moving vertex operators could fail to satisfy the correct quantization conditions [49,50].) The supersymmmetry transformations generate superspace translations along $\hat{\theta}$, and we will be studying antichiral superfields with respect to this supersymmetry, to match the expansion in (2.1). If the Kähler moduli $t^{a}$ are the real parts of scalar components $\phi^{a}$ of term of $N=1$ superfields

$$
\Phi^{a}=\phi^{a}+\hat{\bar{\theta}}^{a}+\hat{\bar{\theta}}^{2} \hat{\bar{y}}^{a}
$$

then a superpotential of the form $W=\phi^{a} \Phi^{b} \Phi^{b}$ leads to terms of the form $\phi^{a} \phi^{b} \hat{\bar{y}}^{c}$ in the low-energy action, where $\hat{\bar{y}}^{i}$ is the auxiliary field. This term exists if the worldsheet correlator

$$
A=\left\langle V_{\phi^{a}}^{(-1,-1)} V_{\phi^{b}}^{(-1,-1)} V_{\hat{\bar{y}}^{c}}^{(0,0)}\right\rangle
$$

where the superscripts refer to the superconformal ghost charge (or, the picture of the vertex operator), and the subscripts to the corresponding spacetime fields. The zeromomentum $(-1,-1)$ picture scalar vertex operators are given in (3.5), and $V_{\hat{\bar{y}}}$ is shown in $(3.10)$.

Therefore, there are six total worldsheet fermions appearing in (6.2). However, in the one-instanton sector of two-dimensional $\mathcal{N}=(2,2)$ theories, there are eight fermion zero modes [78,79]: four left-moving fermions, two with holomorphic spacetime indices and two with antiholomorphic target space indices; and four right-moving fermions, again, two with holomorphic spacetime indices and two with antiholomorphic spacetime indices.

Now imagine that we can slightly deform the Calabi-Yau metric such that the new metric has torsion of the type $W_{1,2}$. In particular imagine giving an expectation value to $y$ in (2.1). This means that the worldsheet action will contain a term of the form $\int d^{2} z V_{y}^{(0,0)}$, with $V_{y}^{(0,0)}$ given in (3.9). In a noncompact model it may be possible to keep the coefficient small (in a compact model we might expect some kind of quantization, as is the case with NS-NS flux, making it difficult to treat the torsion perturbatively). To first order in this perturbation, the cubic term in the superpotential will be nonvanishing if the correlator

$$
A_{1}=\left\langle V_{\phi^{i}}^{(-1,-1)} V_{\phi^{j}}^{(-1,-1)} V_{\hat{\bar{y}}^{k}}^{(0,0)} \int d^{2} z V_{y}^{(0,0)}\right\rangle
$$


is nonvanishing. The form of the vertex operators indicates that there are terms in $A_{1}$ corresponding to expectation values of eight worldsheet fermions with precisely the right spacetime and worldsheet index structure to soak up the zero modes in the one-worldsheetinstanton sector.

Of course, this computation is at best schematic, and valid for local models. It would be interesting and important to describe worldsheet instantons and their effects directly in compactifications with magnetic flux and $S U(3)$ or $S U(3) \times S U(3)$ structures.

\section{Conclusions}

A principle lesson of this paper is that the string worldsheet provides a powerful organizing principle for mathematical structures that describe $\mathcal{N}=1$ type II string backgrounds with intrinsic torsion. Furthermore, it is a necessary organizing tool, since generically such backgrounds will have string-scale features such as nongeometric fluxes, and physical quantities will have contributions that are nonperturbative in $\alpha^{\prime}$.

However, an additional caveat is that in designing realistic string compactifications, one typically includes Ramond-Ramond fields. In this case, one might have to appeal to a formalism such as the one described in 81.

To our minds, further progress in these directions require the construction of more explicit examples of string compactifications with these features. Such features can arise either classically or be sourced by quantum effects. Some progress on classically stabilized moduli, with auxiliary fields for both hypermultiplets and vector multiplets, has already been made for type IIA vacua [82]. Another possibility would be to $N=(2,1)$ gauged linear sigma models describing backgrounds with flux and torsion, taking the recent, elegant construction 83 for $(0,2)$ models as a starting point.

To see how quantum effects might generate the features described in this paper, we note that superpotentials generated by open string gauge theory effects or by D-instantons will generically depend on the Kähler moduli and on the dilaton. If supersymmetry is broken by such F-terms, the auxiliary fields we have described here should be sourced by quantum effects, or should appear classically as duals via a geometric transition as in [84,85]. In particular, for the scenario described in [86,87], the bulk fields mediating supersymmetry breaking are type IIB RR axions, and we expect that the corresponding F-terms will be of the type described in the paper (or at worst will descend from auxiliary fields in another off-shell description of the hypermultiplets). 
Another direction of work which should be relatively straightforward is the extension to heterotic flux compactifications 885-93. In these cases there are already a small set of interesting examples.

\section{Acknowledgements}

We would like to thank Ruben Minasian for early collaboration on this project, and for many useful discussions. We would also like to thank Allan Adams, Oliver DeWolfe, Mariana Graña, Shamit Kachru, John McGreevy, Howard Schnitzer, Alessandro Tomasiello and Daniel Waldram for helpful discussions. Part of this project was carried out while two of us (A.L. and M.S.) were attending the KITP program in string phenomenology in the fall of 2006. A.L. and B.W. would like to thank the theory group at CEA Saclay for their hospitality during part of this project. A.L. would also like to thank the MIT Center for Theoretical Physics for their generous hospitality at various times over the course of this project. This research was supported in part by the National Science Foundation under Grant No. PHY99-07949. A.L. and T.S. are supported by NSF grant PHY-0331516, by DOE Grant No. DE-FG02-92ER40706, and by a DOE Outstanding Junior Investigator award. M.B.S. is supported in part by DOE grant DE-FG02-95ER40893. B.W. is supported in part by NSF grant PHY-00-96515, and by the Frank and Peggy Taplin Membership at the Institute for Advanced Study.

\section{Appendix A. Mathematical conventions}

The $S U(3)$ and $S U(3) \times S U(3)$ structures employed in this paper can be defined, equivalently, in terms of spinors $\eta_{ \pm}^{N}$ or in terms of the differential forms $J^{N}$ and $\Omega^{N}$, for $N=1,2$. The relationship between the two is given in Eqs. (2.8),(2.9) and (2.16). The conventions given below ensure the consistency of these definitions, and are used to compute the auxiliary fields in terms of the intrinsic torsion classes.

\section{A.1. Spinor conventions}

The gamma matrices $\gamma^{A}$, for $A=1, \ldots, 6$ are $8 \times 8$ complex matrices representing the

Clifford algebra $\left\{\gamma^{A}, \gamma^{B}\right\}=2 \eta^{A B}$ where $\eta^{A B}$ is the flat metric in the vielbein basis. The 
gamma matrices with spacetime indices are $\gamma_{m}=e_{m}^{A} \gamma_{A}$, where $e_{m}^{A}$ is the vielbein for the six-dimensional Euclidean compactification manifolds $M$. The gamma matrices

$$
\gamma_{A_{1} \ldots A_{k}}=\gamma_{\left[A_{1}\right.} \gamma_{A_{2}} \ldots \gamma_{\left.A_{k}\right]}
$$

including the identity matrix (for $k=0$ ) and the chirality operator

$$
\gamma_{7}=i \gamma_{123456}
$$

(for $k=6$ ) are all linearly independent. Note that for the Euclidean space $M$ we define $\gamma_{7}$ in (A.2) with a factor of $i$ so that $\left(\gamma_{7}\right)^{2}=1$.

The chiral spinors $\eta_{ \pm}$satisfy the conditions

$$
\begin{aligned}
& \frac{1}{2}\left(1 \pm \gamma_{7}\right) \eta_{ \pm}=\eta_{ \pm}, \\
& \frac{1}{2}\left(1 \mp \gamma_{7}\right) \eta_{ \pm}=0,
\end{aligned}
$$

as well as the Fierz identity

$$
\eta_{ \pm} \otimes \eta_{+}^{\dagger}=\frac{1}{8} \sum_{k=0}^{6} \frac{1}{k !} \eta_{+}^{\dagger} \gamma_{A_{1} \ldots A_{k}} \eta_{ \pm} \gamma^{A_{k} \ldots A_{1}}
$$

where the gamma matrix for the $k=0$ term is the identity matrix. Note that this differs by a factor of 2 from the conventions in Refs. [13, 10]. The factor of $1 / 8$ is fixed by taking the trace of both sides; the trace over the identity matrix on the right hand side gives a factor of 8 since the gamma matrices are $8 \times 8$ matrices if they are to act on spinors of both chiralities.20

It is also useful to define gamma matrices with complex indices, since we use these extensively in this work. As usual, one can define $\gamma^{i}$ and $\gamma^{\bar{\imath}}$ to have the anticommutators

$$
\left\{\gamma^{i}, \gamma^{\bar{\jmath}}\right\}=2 g^{i \bar{\jmath}} \quad \text { and } \quad\left\{\gamma^{i}, \gamma^{j}\right\}=\left\{\gamma^{\bar{\imath}}, \gamma^{\bar{\jmath}}\right\}=0 .
$$

These matrices act as fermionic raising and lowering operators, and can be used to build the spinor representations of $\operatorname{Spin}(6)$. As a consequence of Eqs. (2.16), the spinors $\eta_{ \pm}^{N}$ of Eq. (2.3) satisfy $\gamma^{\bar{i}} \eta_{-}^{N}=\gamma^{i} \eta_{+}^{N}=0$.

20 We would like to thank A. Tomasiello for explaining this, and for his patient and generous help with sorting out the conventions. 


\section{A.2. Metrics, $p$-forms, and the almost complex structure}

Recall that the components of $p$-forms are defined as:

$$
\begin{aligned}
A & =\frac{1}{p !} A_{n_{1} \ldots n_{p}} d x^{n_{1}} \wedge \ldots \wedge d x^{n_{p}} \\
& =\frac{1}{p !} A_{B_{1} \ldots B_{p}} e^{B_{1}} \wedge \ldots \wedge e^{B_{p}}
\end{aligned}
$$

where $e^{B}=e_{m}^{B} d x^{m}$.

As in $\S 3.2$, it is useful to define a complex vielbein $\left\{e^{a}, \epsilon^{\bar{a}}\right\}$ (cf. Eq. (2.12)). Then, in the vielbein basis, the metric is becomes

$$
g_{a \bar{b}}=\frac{1}{2} \eta_{a \bar{b}}
$$

with $\eta_{1 \overline{1}}=\eta_{2 \overline{2}}=\eta_{3 \overline{3}}=1$ and other components of $\eta_{a \bar{b}}$ vanishing. In the same basis, the fundamental form and canonically normalized $(3,0)$ form are

$$
\begin{aligned}
& J=i g_{a \bar{b}} e^{a} \wedge e^{\bar{b}}, \\
& \Omega=\frac{1}{3 !} \epsilon_{a b c} e^{a} \wedge e^{b} \wedge e^{c} .
\end{aligned}
$$

Here, the antisymmetric symbol $\epsilon_{a b c}$ is defined by

$$
\epsilon_{123}=\epsilon_{231}=\epsilon_{312}=1, \quad \epsilon_{213}=\epsilon_{321}=\epsilon_{132}=-1,
$$

with $\epsilon_{a b c}=0$ otherwise. Similarly, we define $\epsilon^{\bar{a} \bar{b} \bar{c}}$ by

$$
\epsilon^{\overline{1} \overline{2} \overline{3}}=\epsilon^{\overline{2} \overline{3} \overline{1}}=\epsilon^{\overline{3} \overline{1} \overline{2}}=1, \quad \epsilon^{\overline{2} \overline{1} \overline{3}}=\epsilon^{\overline{3} \overline{2} \overline{1}}=\epsilon^{\overline{1} \overline{3} \overline{2}}=-1,
$$

with $\epsilon^{\bar{a} \bar{b} \bar{c}}=0$ otherwise. In terms of the latter,

$$
\begin{aligned}
\Omega^{\bar{a} \bar{b} \bar{c}} & =g^{a \bar{a}} g^{b \bar{b}} g^{c \bar{c}} \Omega_{a b c} \\
& =g^{a \bar{a}} g^{b \bar{b}} g^{c \bar{c}} \epsilon_{a b c} \\
& =8 \epsilon^{\bar{a} \bar{b} \bar{c}}
\end{aligned}
$$

For the three-form field strength $H$, we follow the conventions of Polchinski [94, 75 :

$$
H_{m n p}=\partial_{m} B_{n p}+\partial_{n} B_{p m}+\partial_{p} B_{m n},
$$

which is equivalent to $H=d B$, with the forms normalized in terms of their components as in A.6. 


\section{References}

[1] A. Lawrence and J. McGreevy, "Local string models of soft supersymmetry breaking," JHEP 0406, 007 (2004) arXiv:hep-th/0401034.

[2] A. Lawrence and J. McGreevy, "Remarks on branes, fluxes, and soft SUSY breaking," arXiv:hep-th/0401233.

[3] L. Girardello and M. T. Grisaru, "Soft Breaking Of Supersymmetry," Nucl. Phys. B 194, 65 (1982).

[4] C. Vafa, "Superstrings and topological strings at large N," J. Math. Phys. 42, 2798 (2001) arXiv:hep-th/0008142.

[5] S. Gukov, C. Vafa and E. Witten, "CFT's from Calabi-Yau four-folds," Nucl. Phys. B 584, 69 (2000) [Erratum-ibid. B 608, 477 (2001)] arXiv:hep-th/9906070].

[6] T. R. Taylor and C. Vafa, "RR flux on Calabi-Yau and partial supersymmetry breaking," Phys. Lett. B 474, 130 (2000) arXiv:hep-th/9912152].

[7] J. Michelson, "Compactifications of type IIB strings to four dimensions with nontrivial classical potential," Nucl. Phys. B 495, 127 (1997) arXiv:hep-th/9610151.

[8] S. Choissi and S. Salamon, "The Intrinsic Torsion of SU(3) and $G_{2}$ structures," arxiv:math.DG/0202282

[9] S. Gurrieri, J. Louis, A. Micu and D. Waldram, "Mirror symmetry in generalized Calabi-Yau compactifications," Nucl. Phys. B 654, 61 (2003) arXiv:hep-th/0211102.

[10] M. Graña, J. Louis and D. Waldram, "Hitchin functionals in N = 2 supergravity," JHEP 0601, 008 (2006) arXiv:hep-th/0505264.

[11] M. Graña, R. Minasian, M. Petrini and A. Tomasiello, "Generalized structures of N = 1 vacua," JHEP 0511, 020 (2005) [arXiv:hep-th/0505212].

[12] M. Graña, R. Minasian, M. Petrini and A. Tomasiello, "Type II strings and generalized Calabi-Yau manifolds," Comptes Rendus Physique 5, 979 (2004) arXiv:hepth/0409176].

[13] M. Graña, R. Minasian, M. Petrini and A. Tomasiello, "Supersymmetric backgrounds from generalized Calabi-Yau manifolds," JHEP 0408, 046 (2004) arXiv:hepth/0406137.

[14] S. Gurrieri and A. Micu, "Type IIB theory on half-flat manifolds," Class. Quant. Grav. 20, 2181 (2003) arXiv:hep-th/0212278.

[15] S. Fidanza, R. Minasian and A. Tomasiello, "Mirror symmetric SU(3)-structure manifolds with NS fluxes," Commun. Math. Phys. 254, 401 (2005) [arXiv:hep-th/0311122].

[16] A. Tomasiello, "Topological mirror symmetry with fluxes," JHEP 0506, 067 (2005) arXiv:hep-th/0502148.

[17] S. Gurrieri, A. Lukas and A. Micu, "Heterotic on half-flat," Phys. Rev. D 70, 126009 (2004) arXiv:hep-th/0408121. 
[18] M. Graña, J. Louis and D. Waldram, "SU(3) x SU(3) compactification and mirror duals of magnetic fluxes," JHEP 0704, 101 (2007) arXiv:hep-th/0612237.

[19] J. Shelton, W. Taylor and B. Wecht, "Nongeometric flux compactifications," JHEP 0510, 085 (2005) arXiv:hep-th/0508133.

[20] J. Shelton, W. Taylor and B. Wecht, "Generalized flux vacua," JHEP 0702, 095 (2007) arXiv:hep-th/0607015.

[21] B. Wecht, "Lectures on Nongeometric Flux Compactifications," arXiv:0708.3984 [hepth].

[22] G. Aldazabal, P. G. Camara, A. Font and L. E. Ibanez, "More dual fluxes and moduli fixing," JHEP 0605, 070 (2006) arXiv:hep-th/0602089].

[23] I. Benmachiche and T. W. Grimm, "Generalized $\mathrm{N}=1$ orientifold compactifications and the Hitchin functionals," Nucl. Phys. B 748, 200 (2006) arXiv:hep-th/0602241.

[24] A. Micu, E. Palti and G. Tasinato, "Towards Minkowski vacua in type II string compactifications," JHEP 0703, 104 (2007) arXiv:hep-th/0701173].

[25] S. Kachru, M. B. Schulz, P. K. Tripathy and S. P. Trivedi, "New supersymmetric string compactifications," JHEP 0303, 061 (2003) arXiv:hep-th/0211182.

[26] A. Dabholkar and C. Hull, "Duality twists, orbifolds, and fluxes," JHEP 0309, 054 (2003) arXiv:hep-th/0210209.

[27] A. Dabholkar and C. Hull, "Generalised T-duality and non-geometric backgrounds," arXiv:hep-th/0512005.

[28] C. M. Hull and R. A. Reid-Edwards, "Flux compactifications of string theory on twisted tori," arXiv:hep-th/0503114.

[29] J. Gray and E. Hackett-Jones, "On T-folds, G-structures and supersymmetry," arXiv:hep-th/0506092.

[30] S. Hellerman, J. McGreevy and B. Williams, "Geometric constructions of nongeometric string theories," JHEP 0401, 024 (2004) arXiv:hep-th/0208174.

[31] A. Flournoy and B. Williams, "Nongeometry, duality twists, and the worldsheet," arXiv:hep-th/0511126.

[32] S. Hellerman and J. Walcher, "Worldsheet CFTs for flat monodrofolds," arXiv:hepth/0604191.

[33] E. Silverstein, "TASI / PiTP / ISS lectures on moduli and microphysics," arXiv:hepth/0405068.

[34] A. Maloney, E. Silverstein and A. Strominger, "De Sitter space in noncritical string theory," arXiv:hep-th/0205316.

[35] E. Silverstein, "(A)dS backgrounds from asymmetric orientifolds," arXiv:hep-th/0106209.

[36] K. Becker, M. Becker, C. Vafa and J. Walcher, "Moduli stabilization in non-geometric backgrounds," Nucl. Phys. B 770, 1 (2007) arXiv:hep-th/0611001.

[37] S. Kachru, X. Liu, M. B. Schulz and S. P. Trivedi, "Supersymmetry changing bubbles in string theory," JHEP 0305, 014 (2003) arXiv:hep-th/0205108. 
[38] A. K. Kashani-Poor and R. Minasian, "Towards reduction of type II theories on SU(3) structure manifolds," JHEP 0703, 109 (2007) arXiv:hep-th/0611106.

[39] R. Grimm, M. Sohnius and J. Wess, "Extended Supersymmetry And Gauge Theories," Nucl. Phys. B 133, 275 (1978).

[40] B. de Wit and J. W. van Holten, "Multiplets Of Linearized SO(2) Supergravity," Nucl. Phys. B 155, 530 (1979).

[41] M. de Roo, J. W. van Holten, B. de Wit and A. Van Proeyen, "Chiral Superfields In N=2 Supergravity," Nucl. Phys. B 173, 175 (1980).

[42] N. Berkovits and W. Siegel, "Superspace Effective Actions for 4D Compactifications of Heterotic and Type II Superstrings," Nucl. Phys. B 462, 213 (1996) arXiv:hepth/9510106].

[43] O. DeWolfe and S. B. Giddings, "Scales and hierarchies in warped compactifications and brane worlds," Phys. Rev. D 67, 066008 (2003) arXiv:hep-th/0208123.

[44] S. Kachru, J. McGreevy and P. Svrcek, "Bounds on masses of bulk fields in string compactifications," JHEP 0604, 023 (2006) arXiv:hep-th/0601111.

[45] P. Horava and E. Witten, "Heterotic and type I string dynamics from eleven dimensions," Nucl. Phys. B 460, 506 (1996) arXiv:hep-th/9510209.

[46] P. Horava and E. Witten, "Eleven-Dimensional Supergravity on a Manifold with Boundary," Nucl. Phys. B 475, 94 (1996) arXiv:hep-th/9603142.

[47] T. Banks, "Remarks on M theoretic cosmology," arXiv:hep-th/9906126.

[48] T. Banks, "M-theory and cosmology," arXiv:hep-th/9911067.

[49] T. Banks, L. J. Dixon, D. Friedan and E. J. Martinec, "Phenomenology and conformal field theory or can string theory predict the weak mixing angle?," Nucl. Phys. B 299, 613 (1988).

[50] T. Banks and L. J. Dixon, "Constraints on string vacua with spacetime supersymmetry," Nucl. Phys. B 307, 93 (1988).

[51] U. Lindstrom and M. Rocek, "New hyperkahler metrics and new supermultiplets," Commun. Math. Phys. 115, 21 (1988).

[52] M. Graña, "MSSM parameters from supergravity backgrounds," Phys. Rev. D 67, 066006 (2003) arXiv:hep-th/0209200.

[53] P. G. Camara, L. E. Ibanez and A. M. Uranga, "Flux-induced SUSY-breaking soft terms," Nucl. Phys. B 689, 195 (2004) arXiv:hep-th/0311241.

[54] J. M. Maldacena and C. Nunez, "Towards the large N limit of pure $N=1$ super Yang Mills," Phys. Rev. Lett. 86, 588 (2001) arXiv:hep-th/0008001.

[55] A. H. Chamseddine and M. S. Volkov, "Non-Abelian solitons in N = 4 gauged supergravity and leading order string theory," Phys. Rev. D 57, 6242 (1998) arXiv:hepth/9711181].

[56] N. Hitchin, "Generalized Calabi-Yau manifolds," Quart. J. Math. Oxford Ser. 54, 281 (2003) arXiv:math.dg/0209099. 
[57] M. Gualtieri, "Generalized complex geometry", arXiv:math.DG/0401221.

[58] C. M. Hull, "A geometry for non-geometric string backgrounds," JHEP 0510, 065 (2005) arXiv:hep-th/0406102.

[59] A. Lawrence, M. B. Schulz and B. Wecht, "D-branes in nongeometric backgrounds," JHEP 0607, 038 (2006) arXiv:hep-th/0602025.

[60] S. J. Gates, C. M. Hull and M. Rocek, "Twisted Multiplets And New Supersymmetric Nonlinear Sigma Models," Nucl. Phys. B 248, 157 (1984).

[61] J. de Boer, A. Kashani-Poor, and S. El-Showk, to appear.

[62] M. R. Douglas, "D-branes, categories and N = 1 supersymmetry," J. Math. Phys. 42, 2818 (2001) arXiv:hep-th/0011017.

[63] P. G. Camara and M. Graña, "No-scale supersymmetry breaking vacua and soft terms with torsion," arXiv:0710.4577 [hep-th].

[64] C. M. Hull, "Sigma Model Beta Functions And String Compactifications," Nucl. Phys. B 267, 266 (1986).

[65] J. J. Atick, L. J. Dixon and A. Sen, "String Calculation Of Fayet-Iliopoulos D Terms In Arbitrary Supersymmetric Compactifications," Nucl. Phys. B 292, 109 (1987).

[66] M. Dine, I. Ichinose and N. Seiberg, "F Terms And D Terms In String Theory," Nucl. Phys. B 293, 253 (1987).

[67] A. Lawrence and A. Sever, "Scattering of twist fields from D-branes and orientifolds," arXiv:0706.3199 [hep-th].

[68] L. Alvarez-Gaumé, D. Z. Freedman and S. Mukhi, "The Background Field Method And The Ultraviolet Structure Of The Supersymmetric Nonlinear Sigma Model," Annals Phys. 134, 85 (1981).

[69] A. Flournoy, B. Wecht and B. Williams, "Constructing nongeometric vacua in string theory," Nucl. Phys. B 706, 127 (2005) arXiv:hep-th/0404217].

[70] M. Graña, "Flux compactifications in string theory: A comprehensive review," Phys. Rept. 423, 91 (2006) arXiv:hep-th/0509003.

[71] A. Strominger, S. T. Yau and E. Zaslow, "Mirror symmetry is T-duality," Nucl. Phys. B 479, 243 (1996) arXiv:hep-th/9606040.

[72] D. R. Morrison, "Geometric aspects of mirror symmetry," arXiv:math/0007090.

[73] S. Kachru, M. B. Schulz and S. Trivedi, "Moduli stabilization from fluxes in a simple IIB orientifold," JHEP 0310, 007 (2003) arXiv:hep-th/0201028.

[74] M. Graña, R. Minasian, M. Petrini and A. Tomasiello, "A scan for new N=1 vacua on twisted tori," JHEP 0705, 031 (2007) arXiv:hep-th/0609124.

[75] J. Polchinski, "String theory. Vol. 2: Superstring theory and beyond," Cambridge, UK: Univ. Pr. (1998) $531 p$

[76] S. F. Hassan, "T-duality, space-time spinors and R-R fields in curved backgrounds," Nucl. Phys. B 568, 145 (2000) arXiv:hep-th/9907152. 
[77] F. Denef, M. R. Douglas, B. Florea, A. Grassi and S. Kachru, "Fixing all moduli in a simple F-theory compactification," Adv. Theor. Math. Phys. 9, 861 (2005) arXiv:hepth/0503124.

[78] M. Dine, N. Seiberg, X. G. Wen and E. Witten, "Nonperturbative effects on the string worldsheet," Nucl. Phys. B 278, 769 (1986).

[79] M. Dine, N. Seiberg, X. G. Wen and E. Witten, "Nonperturbative effects on the string worldsheet. 2," Nucl. Phys. B 289, 319 (1987).

[80] S. Kachru, S. Katz, A. E. Lawrence and J. McGreevy, "Open string instantons and superpotentials," Phys. Rev. D 62, 026001 (2000) arXiv:hep-th/9912151].

[81] N. Berkovits, "Covariant quantization of the Green-Schwarz superstring in a CalabiYau background," Nucl. Phys. B 431, 258 (1994) arXiv:hep-th/9404162.

[82] O. DeWolfe, A. Giryavets, S. Kachru and W. Taylor, "Type IIA moduli stabilization," JHEP 0507, 066 (2005) arXiv:hep-th/0505160.

[83] A. Adams, M. Ernebjerg and J. M. Lapan, "Linear models for flux vacua," arXiv:hepth/0611084.

[84] M. Aganagic, C. Beem and S. Kachru, "Geometric Transitions and Dynamical SUSY Breaking," arXiv:0709.4277 [hep-th].

[85] M. Aganagic and C. Beem, "Geometric Transitions and D-Term SUSY Breaking," arXiv:0711.0385 [hep-th].

[86] R. Dermisek, H. Verlinde and L. T. Wang, "Hypercharged Anomaly Mediation," arXiv:0711.3211 [hep-ph].

[87] H. Verlinde, L. T. Wang, M. Wijnholt and I. Yavin, "A Higher Form (of) Mediation," arXiv:0711.3214 [hep-th].

[88] K. Becker, M. Becker, K. Dasgupta and P. S. Green, "Compactifications of heterotic theory on non-Kaehler complex manifolds. I," JHEP 0304, 007 (2003) arXiv:hepth/0301161].

[89] K. Becker, M. Becker, P. S. Green, K. Dasgupta and E. Sharpe, "Compactifications of heterotic strings on non-Kaehler complex manifolds. II," Nucl. Phys. B 678, 19 (2004) arXiv:hep-th/0310058.

[90] M. Becker, K. Dasgupta, A. Knauf and R. Tatar, "Geometric transitions, flops and non-Kaehler manifolds. I," Nucl. Phys. B 702, 207 (2004) arXiv:hep-th/0403288.

[91] S. Alexander, K. Becker, M. Becker, K. Dasgupta, A. Knauf and R. Tatar, "In the realm of the geometric transitions," Nucl. Phys. B 704, 231 (2005) arXiv:hepth/0408192].

[92] K. Becker, M. Becker, K. Dasgupta and R. Tatar, "Geometric transitions, non-Kaehler geometries and string vacua," Int. J. Mod. Phys. A 20, 3442 (2005) arXiv:hepth/0411039. 
[93] M. Becker, K. Dasgupta, S. H. Katz, A. Knauf and R. Tatar, "Geometric transitions, flops and non-Kaehler manifolds. II," Nucl. Phys. B 738, 124 (2006) arXiv:hepth/0511099].

[94] J. Polchinski, "String theory. Vol. 1: An introduction to the bosonic string," Cambridge, UK: Univ. Pr. (1998) $402 p$ 